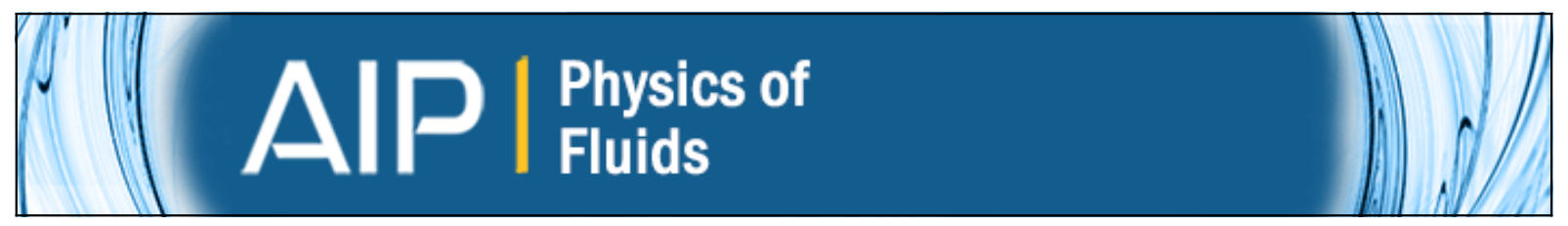

\title{
Reshocked Richtmyer-Meshkov instability: Numerical study and modeling of random multi-mode experiments
}

G. Malamud, E. Leinov, O. Sadot, Y. Elbaz, G. Ben-Dor, and D. Shvarts

Citation: Physics of Fluids (1994-present) 26, 084107 (2014); doi: 10.1063/1.4893678

View online: http://dx.doi.org/10.1063/1.4893678

View Table of Contents: http://scitation.aip.org/content/aip/journal/pof2/26/8?ver=pdfcov

Published by the AIP Publishing

\section{Articles you may be interested in}

An experimental and numerical investigation of the dependency on the initial conditions of the RichtmyerMeshkov instability

Phys. Fluids 26, 024109 (2014); 10.1063/1.4865836

Ejecta source model based on the nonlinear Richtmyer-Meshkov instability

J. Appl. Phys. 113, 024905 (2013); 10.1063/1.4773575

Experimental study of initial condition dependence on Richtmyer-Meshkov instability in the presence of reshock Phys. Fluids 24, 034103 (2012); 10.1063/1.3693152

Richtmyer-Meshkov instability induced by shock-bubble interaction: Numerical and analytical studies with experimental validation

Phys. Fluids 18, 036102 (2006); 10.1063/1.2185685

Experimental study of Richtmyer-Meshkov instability induced by cylindrical shock waves

Phys. Fluids 17, 084101 (2005); 10.1063/1.1964916

\section{Did your publisher get}

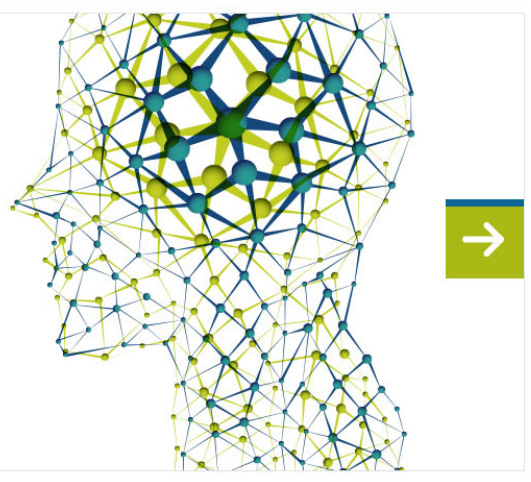




\title{
Reshocked Richtmyer-Meshkov instability: Numerical study and modeling of random multi-mode experiments
}

\author{
G. Malamud, ${ }^{1,2,3, a)}$ E. Leinov, ${ }^{4}$ O. Sadot, ${ }^{2}$ Y. Elbaz, ${ }^{1,5}$ G. Ben-Dor, ${ }^{1}$ \\ and D. Shvarts $1,2,5$ \\ ${ }^{1}$ Department of Physics, Nuclear Research Center - Negev, Beer-Sheva 84190, Israel \\ ${ }^{2}$ Department of Mechanical Engineering, Ben-Gurion University of the Negev, \\ Beer-Sheva 84015, Israel \\ ${ }^{3}$ Department of Aerospace, Oceanic and Space Sciences, University of Michigan, \\ Ann Arbor, Michigan 48109, USA \\ ${ }^{4}$ Department of Mechanical Engineering, Imperial College London, SW7 2AZ London, \\ United Kingdom \\ ${ }^{5}$ Department of Physics, Ben-Gurion University of the Negev, Beer-Sheva 84015, Israel
}

(Received 3 February 2014; accepted 11 August 2014; published online 26 August 2014)

\begin{abstract}
The evolution of the three-dimensional planar Richtmyer-Meshkov (RM) instability during a two shock wave interaction (i.e., reshock) is investigated by means of comparing numerical simulations and analytical modelling with experimental results of low Mach numbers $(M<1.5)$ and fairly high Atwood numbers $(A \sim 0.7)$. The study discusses and analyses the differences in the evolution of the mixing zone for two different types of initial perturbations, namely, multi-mode random initial perturbation with a narrow or wide bubble size distribution. More specifically, the study is focused on the agreement between numerical simulations and experiments performed with an unknown random initial perturbation. Using a large set of experimental results with different reshock arrival times and Mach numbers, the numerical simulations results are compared to the experimental results for a variety of different scenarios. This methodology allows a constrained comparison, while requiring good agreement for all cases. A comprehensive parametric study is conducted, examining the evolution of the mixing zone (MZ) for different initial amplitudes and wavelengths. It is found that in order to achieve a good agreement, the numerical simulation must be performed using a wide enough initial spectrum, which enables a dominant, efficient bubble merging process to take place within the MZ. The numerical simulation results are compared to a model, based on classic single bubble RM evolution formulation, combined with high amplitude effects consideration and phase reversal treatment in case of heavy to light reshock passage. The model is also extended for the case of multi-mode fronts, accounting for a bubble merging process, determining that the MZ evolution after the reshock can be classified with high confidence as governed by an inverse cascade bubble merger, approaching self-similarity. @ 2014 AIP Publishing LLC. [http://dx.doi.org/10.1063/1.4893678]
\end{abstract}

\section{INTRODUCTION}

The Richtmyer-Meshkov (RM) instability ${ }^{1,2}$ occurs when a shock wave passes an interface separating two fluids of different densities, causing small perturbations initially present on the interface to grow in the form of light bubbles floating into the heavy fluid and heavy fluid spikes penetrating into the light fluid, eventually forming a mixing zone (MZ). Due to its importance in a variety of scientific and engineering fields such as inertial confinement fusion $\left(\mathrm{ICF}^{3,4}\right)$ and astrophysics (e.g., super-nova ${ }^{5-8}$ ), the RM instability has been investigated extensively in the past

\footnotetext{
a) Author to whom correspondence should be addressed. Electronic mail: gmalamud@umich.edu
} 
decades. In many cases, the interface interacts with more than one shock wave. For example, during the capsule implosion in ICF, the interface interacts first with the ablative shock wave propagating towards the capsule center, and then with the rebounding shock wave (reshock) reflected from the center.

The RM evolution due to a single shock wave interaction was investigated intensively in the past decades for single- and multi-mode initial perturbations, both theoretically, ${ }^{1,9-29}$ and experimentally., ${ }^{2} 30-37$ Many models, providing a comprehensive description of the evolution of a single-mode RM instability for the single-shock case exist in the open literature. The most updated study was presented in Dimonte $e t$ al. ${ }^{28}$ along with a detailed review of existing models, and this case is well understood.

In spite of the experimental studies that were conducted regarding the evolution of the RM instability MZ when it is accelerated by multiple shock waves, ${ }^{38-46}$ the governing mechanisms in this case are not yet fully understood, both for single-mode and the random multi-mode initial perturbations. This is especially true for the latter case, since experimental results often provide only integral data about the MZ, (i.e., the change of its width in time) and do not provide information about the inner small scale structure of the MZ or about the nonlinear interaction between different bubbles or spikes. Moreover, the majority of the experimental data for the multi-mode initial perturbation case was collected in shock tube experiments, where the initial perturbations are formed randomly on a thin membrane, separating the two gases. ${ }^{38-41,45,46}$ For this type of experiments, in which the membrane is randomly ruptured by the shock wave, the possibility of a classical single-mode initial perturbation is small, and the initial perturbation on the interface can be characterized as a random multi-mode one. As a result, the exact initial perturbation's spectrum is unknown, and a comprehensive analysis of the experiments becomes a challenging task. In the case of a wide initial bubble size distribution in the initial perturbation spectrum, the MZ evolution can be typically categorized as dominated by bubble merging dynamics. For a wide enough initial bubble size distribution, the MZ evolution will reach a self-similar regime. ${ }^{26}$ In the extreme case of a very narrow initial distribution, the evolution of the mixing zone will loose the bubble merging mechanism dominance, and a pseudo single-mode regime will govern the $\mathrm{MZ}$ evolution.

When the RM instability MZ experiences a second shock wave (reshock), additional energy is deposited in the mixing process. As a result, the growth of the MZ increases dramatically and the mixing process is enhanced. ${ }^{31,38,39,41-43,45-47}$ The interface shock-reshock interaction is more complex than the single shock interaction, mainly because of two elements: first, when the reshock interacts with the MZ, the perturbation amplitude is usually significantly larger than the small amplitude assumed in most of the present models; second, when the reshock interacting with the MZ passes from a high density to a low density fluid, a complex phase reversal takes place, especially if the MZ amplitude is high. It should be noted that the vast majority of experimental setups used to study reshocks using shock tubes, and therefore the reshock direction was opposite to the direction of the incident shock. Therefore, a phase reversal took place in all the cases of a light/heavy configuration (e.g., air - $\mathrm{SF}_{6}$ was used in many of the experimental studies). ${ }^{31,38,41-43,45,46}$

During the past years, several models were developed in order to grasp the different stages of the reshock process, and to obtain a comprehensive understanding about the nature of the MZ growth. ${ }^{22,40,48,49}$ Unfortunately, all these models are semi-phenomenological, and they assume that the $\mathrm{MZ}$ amplitude remains small throughout the entire process, ${ }^{40}$ that the reshock is fairly weak compared to the incident shock ${ }^{48}$ (which is not the case for heavy to light, air - $\mathrm{SF}_{6}$ shock tube experiments, such as the experiments of Leinov et al. ${ }^{46}$ that are discussed below), or that the model contains one or more free parameters which has to be calibrated. ${ }^{22}$ Moreover, the predictions of these models were recently compared to experimental results ${ }^{46}$ and poor agreement was evident. The best agreement was obtained using the Mikaelian model, ${ }^{22,49}$ which underestimated the growth rate of the MZ after the reshock by about $23 \%$.

There are some numerical studies, where the effect of the initial spectrum on the evolution of the multi-mode RM instability was investigated for one or two shock waves interaction. ${ }^{47,50-53}$ However, the comparisons to experiments were done for a single given configuration (i.e., shock wave Mach number, shock tube dimensions, etc.). Moreover, these studies did not present a comprehensive parametric study of possible initial conditions, which could successfully reproduce the 
experiments. As a result, the uncertainty in the experimental results and the initial conditions was not removed.

In the present study, we present a comprehensive numerical research, in order to better classify the evolution of the MZ under reshock conditions in the experimental results of Leinov et al., ${ }^{46}$ which will be described subsequently. The study is performed over two types of initial multi-mode spectra, which are different in their initial spectral distributions. A large variety of initial conditions is simulated (i.e., initial average wavelengths and amplitudes) and compared to the experimental results, with several shock tube dimensions and shock wave Mach numbers.

Due to the complexity of the full three-dimensional numerical simulations, it is hard to separate the basic mechanisms governing the evolution of the MZ. Hence, an additional goal of the present study is to better understand the evolution of the bubbles' height, both for the single- and multi-mode initial perturbation cases, while using a simple model based on classical, well known RM physics, as was done previously for the single shock case. ${ }^{15,26,28,31}$ Since accurately extracting this data (bubble front position relative to the one-dimensional surface position) from the available experimental results is difficult due to experimental uncertainties, the study will rely on the numerical simulations when evaluating the model.

The methodology used in the present study is presented subsequently following the presentation of the experimental results of Leinov et al. ${ }^{46}$ In Sec. II we present a general numerical analysis of the evolution of the reshocked MZ in the single- and multi-mode cases. In Sec. III the comparison of the numerical simulations with the experiments is discussed. Finally, the present model is described in Sec. IV, along with an analysis of the MZ evolution in the reshock case for the experiments and simulations at hand.

\section{A. The experiments of Leinov et al. ${ }^{46}$}

Leinov et al. ${ }^{46}$ presented a comprehensive experimental study of the reshock effect on the MZ growth rate and amplitude. The study was conducted using a square shock tube, having an $80 \mathrm{~mm} \times$ $80 \mathrm{~mm}$ cross section. The research was done in a light/heavy configuration, using air and $\mathrm{SF}_{6}$ as the light and heavy fluids, respectively $(A \approx 0.67)$. The two gases were separated in the test-section of the shock tube using a thin membrane having randomly distributed small scale perturbations, which have random wavelengths ranging up to $\lambda \sim 1 \mathrm{~mm}$. The upper limit of the wavelength (i.e., $\lambda \sim 1 \mathrm{~mm}$ ) was established by collecting and analyzing the membrane fragments after each experiment. However, this post-shot analysis does not mean that larger fragments (i.e., larger wavelengths) were not present at the beginning of the experiments. Therefore, in order to cover this point, the numerical parametric study of the initial conditions (see Sec. I B) included larger wavelengths as well. Upon the rupture of this membrane by the shock wave, the mixing process commenced. As mentioned in Sec. I, a perfect single-mode initial perturbation is highly unlikely due to the random nature of the perturbations on the membrane edges, and the growth of the MZ is expected to be multimode governed. The length of the test-section was determined by positioning the rigid end-wall at a location of 80-235 $\mathrm{mm}$ downstream of the membrane. The experiments were recorded using a schlieren diagnostic system with high-speed rotating-drum camera having time resolution of $56 \mu \mathrm{s}$. The experiments explored the reshock effect on the MZ growth in two aspects:

- The dependence of the post reshock MZ growth rate on the MZ amplitude at the reshock arrival. This was achieved by changing the shock tube end-wall position, and as a result allowing the $\mathrm{MZ}$ to grow for a longer time before the reshock arrival.

- The dependence of the post reshock MZ growth rate on the incident shock wave and reshock strengths. This was achieved by changing the incident shock wave Mach number $\left(M_{i}\right)$ that in turn changed the reshock strength as well.

A summary of the experimental cases is given in Table I. The experiments were proven to be highly reproducible, with an experimental measurement error of about $2 \%$ (for further details, see Leinov et $a l .{ }^{46}$ ). For a given end-wall position and incident shock wave Mach number, the experimental dispersion was less than $0.4 \mathrm{~mm}$ over one standard deviation $(1 \sigma)$. The overall difference between the different experiments that were conducted with different end-wall positions and fixed $M_{i}=1.2$ 
TABLE I. MZ width prior to the reshock arrival and post reshock MZ growth rates (for the first $0.5 \mathrm{~ms}$ after the reshock), measured in the experiments of Leinov et al. ${ }^{46}$ for end-wall positions of 80, 172, and $235 \mathrm{~mm}$ and $M_{i}=1.2$, and for an end-wall position of $80 \mathrm{~mm}$, with $M_{i}=1.15$ and 1.3 .

\begin{tabular}{lllccc}
\hline \hline Case & $M_{i}$ & $M_{\text {ref }}$ & $\begin{array}{c}\text { End-wall position } \\
(\mathrm{mm})\end{array}$ & $\begin{array}{c}\text { MZ width at reshock } \\
(\mathrm{mm})\end{array}$ & $\begin{array}{c}\text { MZ growth rate after reshock } \\
(\mathrm{mm} / \mathrm{ms})\end{array}$ \\
\hline 1 & 1.2 & 1.3 & 80 & $4.3 \pm 0.7$ & $23.1 \pm 1.0$ \\
2 & 1.2 & 1.3 & 172 & $6.7 \pm 1.1$ & $23.0 \pm 1.0$ \\
3 & 1.2 & 1.3 & 235 & $6.3 \pm 1.0$ & $23.8 \pm 1.0$ \\
4 & 1.15 & 1.2 & 80 & $3.5 \pm 1.0$ & $13.1 \pm 1.5$ \\
5 & 1.3 & 1.47 & 80 & $5.0 \pm 0.5$ & $34.6 \pm 3.0$ \\
\hline \hline
\end{tabular}

was found to be less than $0.3 \mathrm{~mm}$ in the measured MZ width at the reshock arrival, over the range of two standard deviations, mainly due the uncertainty of \pm 0.02 in the incident shock Mach number.

The post reshock growth rate of the MZ increased dramatically for all the tested configurations (compared to the growth rate prior to reshock passage), reaching a constant growth rate for the first $0.5 \mathrm{~ms}$ after the reshock. It was also found, that the growth rate of the MZ width after the reshock passage was independent of the MZ width prior to its arrival (Cases 1-3 in Table I). The maximal difference in the growth rate after the reshock between the experiments conducted with different end-wall positions was about $3 \%$. The growth rate was found to strongly depend on the reshock Mach number, $M_{\text {ref. }}$. A change of about $25 \%$ in $M_{\text {ref }}$ resulted in a change by a factor of 2.6 in the MZ growth rate (Cases 1, 4, and 5 in Table I).

It was also found that the MZ growth rate after the reshock could be scaled with the reflected shock wave Mach number $M_{r e}$, and that the width evolution of the MZ could be scaled for different $M_{r e f}$ with the distance travelled by the interface $\Delta u t$, where $\Delta u$ is the overall velocity change following the reshock passage. Multi-mode numerical simulations presented in the study ${ }^{46}$ indicated that the MZ dynamics after the reshock passage is dominated by an inverse cascade process of bubble merging, resulting in a large increase of the average wavelength. However, the numerical simulations were done with one specific initial perturbation, and were not meant to check whether other types of initial conditions could reproduce the experimental results.

\section{B. Methodology}

Since the experimental study contained results that were obtained with different initial parameters, which could be used as constraints over the numerical simulations, it could be used to resolve the perturbation dynamics in the experiments. As was discussed in Sec. I A, the breaching of the membrane results with multi-mode initial perturbations. It should be noted again that these initial perturbations are unknown in all the three-dimensional, multi-mode random experiments conducted in shock tubes ${ }^{39-41,45,46}$ or in the LEM system. ${ }^{25}$

In general, bubble merging is expected when the initial condition is a multi-mode one. However, the typical merging rates increase/decrease as the perturbation spectrum width increase/decrease. Therefore, the three-dimensional numerical simulations done in order to resolve the experimental initial condition issue were performed using two sets of possible initial perturbations: multi-mode, with a wide spectrum and multi-mode, with a narrow spectrum. First, the agreement to the experimental results for the two sets of initial perturbations was examined for Case 1 in Table I. A parametric study covering the possible initial conditions range (initial wavelength and amplitude) was performed, and the specific initial condition, which optimally matched the experimental results of Case 1 (over a $2 \sigma$ range) was found for each set. The agreement with the experiments was determined by comparing the experimental MZ width to the numerically simulated one, and the optimal initial condition for each set was chosen as the one resulting with in the minimal divergence from the experiments.

After determining the optimal initial condition to Case 1 of the experiments (end-wall at $80 \mathrm{~mm}$ ) for each set, it was used to reproduce the experimental results under the experimental constraints, 


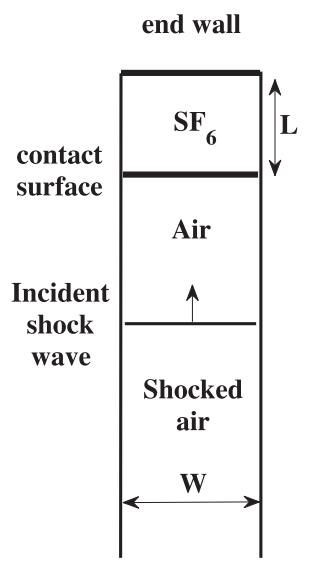

FIG. 1. Schematic illustration of the numerical simulations setup. L - initial distance of the contact surface to end-wall. W domain width, the same in both the $\mathrm{x}$ and the $\mathrm{y}$ directions.

i.e., the experimental results for the end-wall positions of 172 and $235 \mathrm{~mm}$ (Cases 2 and 3 in Table I), and if further needed, the experiments conducted with $M_{i}=1.15$ and 1.3 (Cases 4 and 5 in Table I). An initial condition will be defined as an admissible one only if it reproduces the experimental results under all the available mentioned constraints. Initial conditions for sets which did not result in a good agreement to the experiments were disqualified with high confidence. Naturally, in the case that only one set was found to be in good agreement to all the experiments, it was possible to classify the initial conditions and the flow regime dominating the experiments with high confidence. Finally, the present model was used to better analyze the evolution of the instability.

\section{NUMERICAL ANALYSIS}

The numerical simulations were carried with LEEOR3D, ${ }^{54,55}$ a finite volume ALE (Arbitrary Lagrangian Eulerian) hydrodynamic code with interface tracking capability. ${ }^{56}$ The code solves the Euler equations (viscosity effects are neglected), using the leapfrog method. The Lagrangian scheme used in the code is based on a simple force balance equation for computing the mesh velocities. All the simulations to be presented were initially fully Lagrangian, gradually transferring to fully Eulerian cubic mesh cells in the perturbation region after the incident shock passage.

A schematic drawing of the simulated shock tube setup is presented in Fig. 1. The examined end-wall positions (defined as "L" in Fig. 1) were 80, 172, and $235 \mathrm{~mm}$, as in Leinov et al., ${ }^{46}$ and the $\mathrm{SF}_{6}$ and air properties are given in Table II. The domain width (defined as "W" in Fig. 1) was varied in the simulations according to the requested wavelength. The heights of the fronts of the bubbles and spikes were calculated by taking the $1 \%$ and $99 \%$ edge of the one-dimensional average volume fraction of the air and the $\mathrm{SF}_{6}$, respectively. After the positions of the fronts of the bubbles and spikes

TABLE II. $\mathrm{SF}_{6}$ and air properties (adiabatic constant, $\gamma$, molecular weight, $M$, initial density, $\rho_{0}$, and initial temperature, $T_{0}$ ) used in the numerical simulations.

\begin{tabular}{lcccc}
\hline \hline & & Molecular & Initial & Initial \\
& Adiabatic & weight & density & temperature \\
& constant & $M$ & $\rho_{0}$ & $T_{0}$ \\
& $\gamma$ & $(\mathrm{g} / \mathrm{mole})$ & $\left(\mathrm{g} / \mathrm{cm}^{3}\right)$ & $(\mathrm{K})$ \\
\hline $\mathrm{SF}_{6}$ & 1.1 & 146.04 & $6.2517 \times 10^{-3}$ & 300 \\
Air & 1.4 & 28.95 & $1.225 \times 10^{-3}$ & 300 \\
\hline \hline
\end{tabular}


were obtained, the MZ width was obtained as their difference, and their heights could be calculated relative to the contact surface position. The latter was obtained using a time integral over the contact surface velocity $\Delta u$. It should be noted that in the case of interest (reshock), one has to consider two contact surface velocities, $\Delta u_{1}$ and $\Delta u_{2}$, for the incident shock and reshock, respectively. The boundary conditions used in the simulations were all reflective, except for the upstream side of the domain, where an inflow boundary condition was implemented, in order to describe the mass inflow behind the in incident shock.

\section{A. Single-mode evolution}

Although a simple single-mode initial condition can be ruled out as a possible candidate for the experiments due to the nature of the membrane breaking (see discussion above), it is useful to analyze this relatively simple case first. Also, as will be described further below, an understanding of the single bubble behavior in the reshock case is essential for the building of the model, described in Sec. IV. The single-mode numerical simulations were performed using a numerical resolution of 40 numerical cells per wavelength. In order to reduce the computational time, the simulated domain held only one quarter of the total cross section area, with reflective boundary conditions $(20 \times 20$ numerical cells in the cross section). However, in all the figures to be presented, the total area domain will be shown for clarity. As discussed, the simulations were initially fully Lagrangian, gradually transferring to fully Eulerian cubic mesh cells in the perturbation region while keeping the Lagrangian description of the shock movement down the tube. The transition to a Eulerian mesh was initiated only after the perturbations reached an amplitude to wavelength ratio, $a / \lambda$, of 0.1 , and was done at $a / \lambda \sim 0.2$. In the case of an initial amplitude to wavelength ratio greater than 0.1 , the transitions started after reaching the limit of $\Delta u t>5 \mathrm{~mm}$. Hence, the initial growth of the perturbation was described with a satisfactory precision. The initial perturbation amplitude for the single-mode simulations was defined by a square mode initial perturbation, used also by the alpha group collaboration: ${ }^{.8}$

$$
a_{0}(x, y)=\frac{a_{0}}{2}\left[\cos \left(\phi_{x}\right)+\cos \left(\phi_{y}\right)\right]
$$

where $a_{0}$ on the right-hand side is the initial bubble amplitude and $\phi_{x}=\pi x / W, \phi_{y}=\pi y / W$. We shall discuss now the characteristic evolution of the MZ in a typical simulation, with $\lambda=3 \mathrm{~mm}$, $a_{0} / \lambda=0.03$, and $M_{i}=1.2$, and will present the comparisons to the experiments in Sec. III and to the model predictions in Sec. IV.

Four typical frames from the simulation are presented in Fig. 2. For this setup, the reshock interacts with the contact surface $0.78 \mathrm{~ms}$ after the passage of the incident shock wave. Prior to the reshock arrival, a typical RM instability bubble and spike formation is evident (Fig. 2(a)). The interface is built from one bubble evolving around the centerline $(x=y=0)$, and four spikes, located in the domain corners. During the phase reversal (Fig. 2(b)) the bubble collapses through its center, forming a new spike that evolves towards the negative z-direction (Fig. 2(c)). It is convenient to address the time of minimal amplitude during the phase reversal as a reference point, and therefore, from here on, we shall note this time as $t=0$. The phase reversal completes in about $0.1 \mathrm{~ms}$, with four bubbles evolving in the positive z-direction, and one spike evolving towards the negative z-direction. This formation is maintained until the arrival of the rarefaction wave (Fig. 2(d)). Inspecting Figs. 2(a)-2(c) indicates that the phase reversal occurs through the center of the bubble, while the spikes keep their shape initially and eventually disintegrate. This kind of behavior was previously observed in two-dimensional experiments. ${ }^{34}$ By comparing Figs. 2(a) and 2(c) one can also observe that the interface after the reshock contains small scale structures, indicating an enhanced mixing rate due to a large shear deposition during the passage of the reshock. It should be noted that the use of numerical simulations in the present study was not for describing small scale turbulence that occurs inside the MZ, but only to obtain a physical understanding of the large scale evolution. 
(a) $t=-0.05$



(c) $t=0.1$

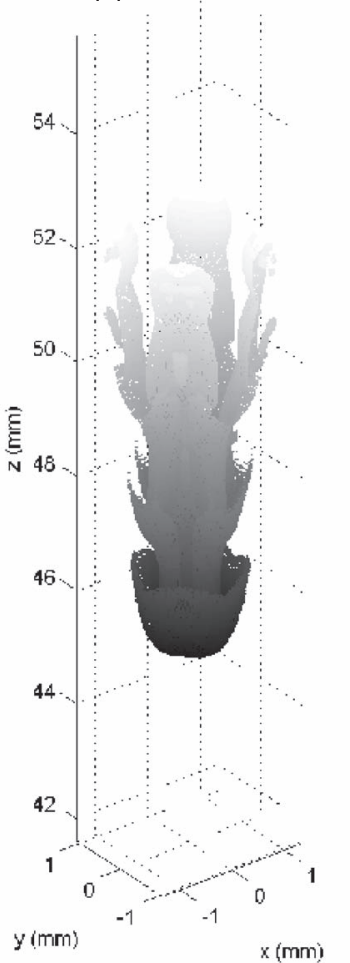

$\mathrm{mm}$
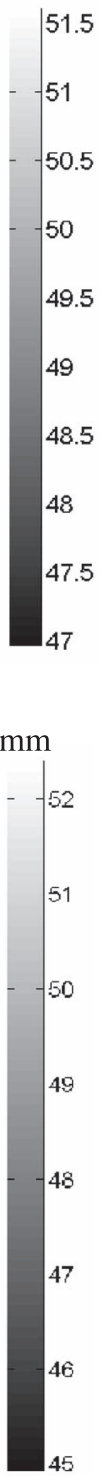

(b) $t=0$

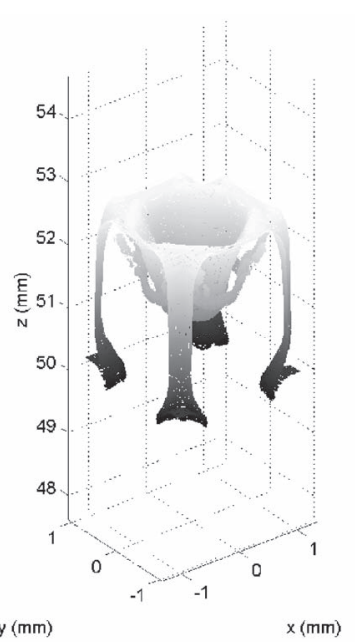

(d) $\mathrm{t}=0.35$

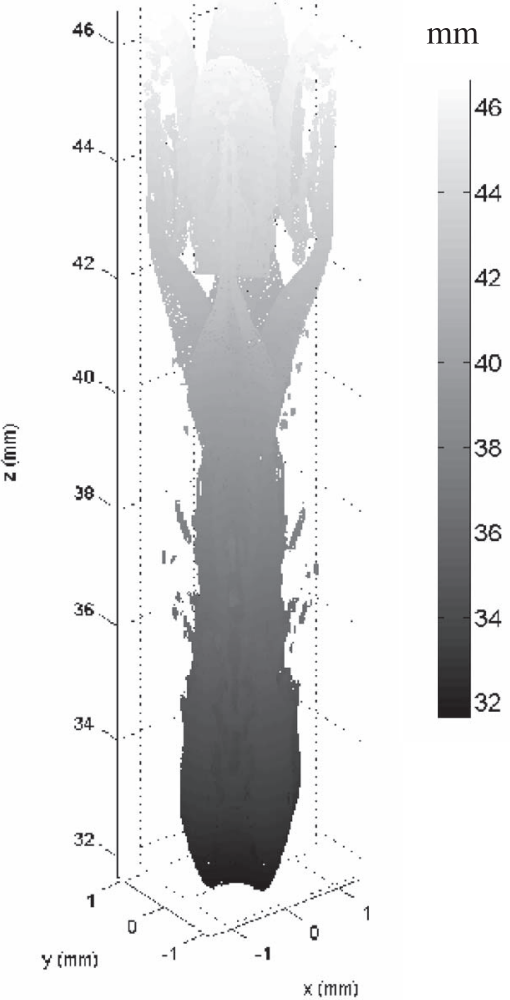

$\mathrm{mm}$


4
38 36

32

FIG. 2. The interface between the air (bottom) and $\mathrm{SF}_{6}$ (top) in four typical frames, in a single-mode numerical simulation with $M_{i}=1.2$ for the incident shock wave, $\lambda=3 \mathrm{~mm}$ and $\mathrm{L}=80 \mathrm{~mm}$. $\mathrm{t}=0$ denotes the minimal MZ amplitude time.

The MZ width and bubble and spike absolute heights as a function of time are presented in Fig. 3. Also presented are the velocities obtained by the linear impulsive model, given by

$$
U_{R M}=A \Delta u k a_{0}^{*}
$$

where $\Delta u$ is the shock induced interface velocity, $k=\sqrt{k_{x}^{2}+k_{y}^{2}}$ is the wave number, $a_{0}^{*}$ is the initial post shock amplitude, and $A=\left(\rho_{2}-\rho_{1}\right) /\left(\rho_{2}+\rho_{1}\right)$ is the post shock Atwood number. 


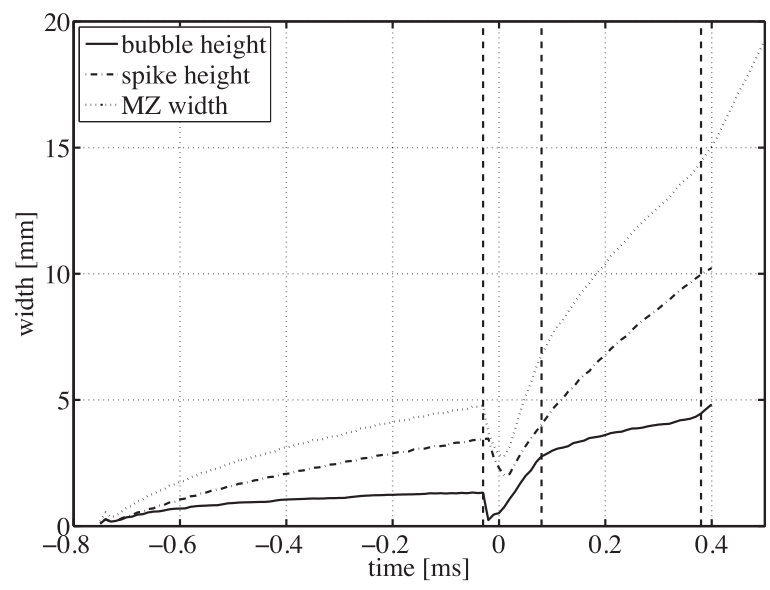

FIG. 3. MZ width (dotted line), bubble height (solid line), and spike height (dashed line) as a function of time, obtained from the 3D numerical simulations with $M_{i}=1.2$ for the incident shock wave, $\lambda=3 \mathrm{~mm}$ and $L=80 \mathrm{~mm}$. The vertical dashed lines represent the times of the reshock interaction with the bubble tip, the end of the phase reversal, and the arrival of the rarefaction wave. $\mathrm{t}=0$ denotes the minimal MZ amplitude time.

Until the arrival of the reshock to the interface $(t<-0.05 \mathrm{~ms})$, the heights of the spike and the bubble evolve according to the classic RM evolution, forming an about 5-mm wide MZ, with spike to bubble amplitude ratio of about 3 (see Fig. 2(a)). When the reshock interacts with the bubble tip, the MZ width and the bubble height decrease sharply, due to the Lagrangian compression and phase reversal (see Fig. 2(b)). When the reshock passes the z-position of the contact surface, the spike amplitude begins to decrease as well, and the bubble amplitude begins to grow. When the new spike evolving from the collapsing bubble completes its formation $(t \sim 0.1 \mathrm{~ms}$, see also Fig. 2(d)), the bubble amplitude continues to grow in a decaying rate until the arrival of the rarefaction, at $t \sim 0.4 \mathrm{~ms}$. The bubble velocity is presented in Fig. 4. It can be seen that after the passage of the incident shock wave $(t=-0.75)$, the bubble velocity reaches the linear velocity $U_{R M}$ (Eq. (2)), marked with horizontal line in the figure, and after that it decays, reaching the asymptotic regime of $u_{b} \sim \lambda / t$. During the phase reversal $(-0.05<t<0.08)$ the velocity reaches a negative value first due to compression and phase reversal, and then it levels out to an approximately constant value $(0<t<0.08)$. When the phase reversal is complete, the velocity decays rapidly, reaching again the asymptotic regime until the arrival of the rarefaction. When comparing the bubble velocity after

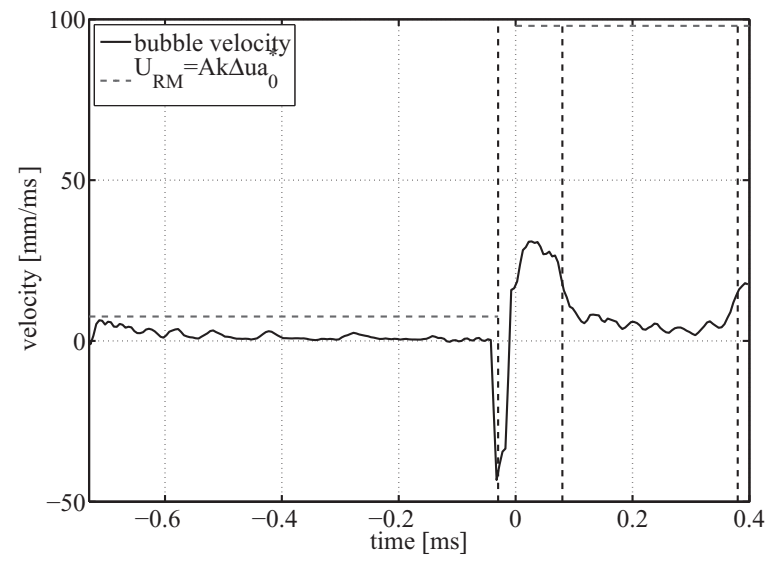

FIG. 4. Bubble velocity as a function of time, obtained from the 3D numerical simulations with $M_{i}=1.2$ for the incident shock wave, $\lambda=3 \mathrm{~mm}$ and $\mathrm{L}=80 \mathrm{~mm}$. The vertical dashed lines represent the times of the reshock interaction with the bubble tip, the end of the phase reversal, and the arrival of the rarefaction wave, respectively, from left. The horizontal blue dashed lines represent the linear velocities $U_{R M}$ before and after the reshock, derived from Eq. (2). 
the reshock to the linear velocity $U_{R M}$ obtained by using Eq. (2) with the proper bubble height, Atwood number, and contact surface overall velocity jump $\left|\Delta u_{2}-\Delta u_{1}\right|$, one finds that the bubble velocity after the arrival of the reshock is about $1 / 3$ of the expected linear value. This effect is related to the fact that upon the interaction of the reshock with the MZ, the bubble amplitude to wavelength ratio reaches a large value of about $1 / 2$, and therefore the bubble initial velocity is decreased.

It can be summarized that the total evolution process can be divided into three stages:

- Pre-reshock stage, in which the classical RM models are valid.

- Phase reversal, governed by the bubble evolution with an approximately constant growth rate.

- Post-reshock phase, in which the growth rate returns to the asymptotic $\sim \lambda / t$ behavior.

\section{B. Multi-mode evolution}

All the multi-mode numerical simulations were performed using a minimum of eight numerical cells per wavelength at $\mathrm{t}=0(128 \times 128$ numerical nodes in a cross section $)$, again starting as fully Lagrangian and then gradually rezoning to cubical mesh cells in the perturbation region. Hence, a satisfactory numerical description of the growing instability was achieved. As was noted, this basic setup was used in an earlier study ${ }^{46}$ to reproduce the experimental results. It is noted that numerical convergence was evident by comparing the numerical simulation results to those obtained in a 256 $\times 256$ simulation with the same initial perturbation. The initial perturbation amplitude for a given spectrum in the numerical simulations was defined by

$$
\begin{aligned}
a_{0}(x, y)=\sum_{k_{x}, k_{y}} & {\left[a_{k} \cos \left(\phi_{x}\right) \cos \left(\phi_{y}\right)\right.} \\
& +b_{k} \cos \left(\phi_{x}\right) \sin \left(\phi_{y}\right) \\
& +c_{k} \sin \left(\phi_{x}\right) \cos \left(\phi_{y}\right) \\
& \left.+d_{k} \sin \left(\phi_{x}\right) \sin \left(\phi_{y}\right)\right],
\end{aligned}
$$

where $\phi_{x}=2 \pi k_{x} x / W, \phi_{y}=2 \pi k_{y} y / W$, and $a_{k}, b_{k}, c_{k}$, and $d_{k}$ are random coefficients. The initial distribution of the wavelength was controlled by the range of the mode numbers, $k_{x}$ and $k_{y}$, and the desired initial amplitude was obtained by multiplying the result with a constant. This definition exhibits in a flat spectrum, having a non-zero amplitude in the range of the chosen mode numbers. Using this, one could define the average wavelength as $\langle\lambda\rangle=\sqrt{S / N}$, where $S$ is the area of the total domain and $N$ is the number of bubbles, initially equal to about 650 in all the simulations. In order to select a desired initial average wavelength for a given initial distribution, the total area was changed while keeping the range of mode numbers. In a similar manner, the characteristic length of the i-th single bubble was defined $\lambda_{i}=\sqrt{S_{i}}$, where $S_{i}$ was calculated using a simple watershed algorithm. ${ }^{57}$ The watershed algorithm is a standard key tool developed within the framework of mathematical morphology for segmenting images. It detects the watershed lines on the bubble front image by detecting the catchment basins of all minima in the gradient image. Hence, by analysing the bubble front, one could obtain the size distribution of the bubbles. It should be noted here that the present definition of the wavelength, $\lambda_{i}$, defined here is not consistent with the classic definition, $k=2 \pi / \lambda$. However, it fairly represents the typical length scales of the bubbles. Finally, the initial average amplitude was defined as the ensemble average $\left\langle a_{0}\right\rangle=\sum_{i=1}^{N}\left(a_{0, i} S_{i}\right) / S$, where $a_{0, i}$ is the $i$ th bubble initial amplitude, defined by its peak's height.

As discussed earlier, the multi-mode numerical simulations were conducted with two different initial distributions, a narrow one and a wide one, defined using Eq. (3). The ranges of the mode numbers $k_{x}$ and $k_{y}$ were 4-32 and 8-16 for the wide and the narrow distributions, respectively. The initial average wavelength was varied for the parametric study by simply increasing or decreasing the domain cross section, $W$. Hence, the study was done while keeping $\Delta \lambda /\left\langle\lambda_{0}\right\rangle$ constant. It should be noted that the numerical simulation results were found to be insensitive to the random factors in Eq. (3). The initial distributions and the corresponding initial contact surfaces for the two distributions 

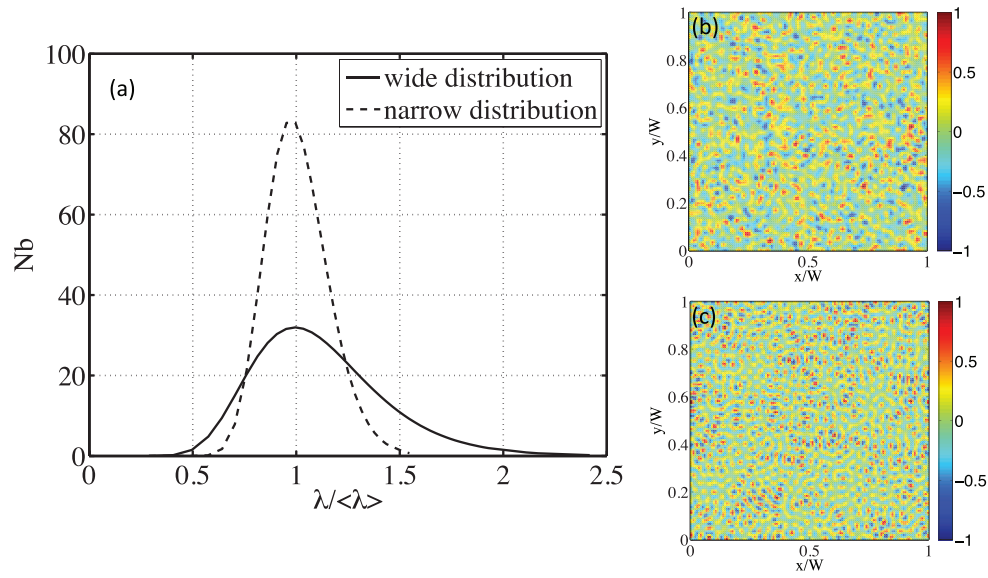

FIG. 5. The initial number of bubbles vs. normalized wavelength (a), and the initial interface between the air and $\mathrm{SF}_{6}$ in arbitrary units, for the wide (b) and narrow (c) initial distributions used in the numerical simulations.

are presented in Fig. 5. It can be seen that the value of $\Delta \lambda /\left\langle\lambda_{0}\right\rangle$ is about 2 and about 1 for the wide and narrow distributions, respectively.

Four typical frames obtained from the simulation of a wide distribution, $M_{i}=1.2,\left\langle\lambda_{0}\right\rangle=0.4 \mathrm{~mm}$, $\left\langle a_{0}^{*}\right\rangle /\left\langle\lambda_{0}\right\rangle=0.2$, and $L=80 \mathrm{~mm}$ are presented in Fig. 6 (note that the frames are not to scale). The number of bubbles is decreasing with time due to bubble merging, ${ }^{15,26}$ both before and after the

(a) $\mathrm{t}=-0.05$

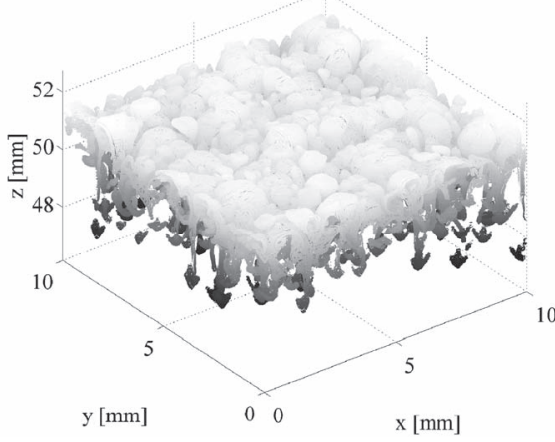

(c) $t=0.1$

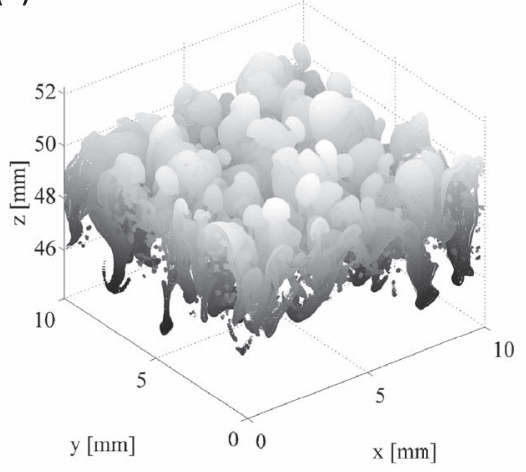

(b) $t=0$
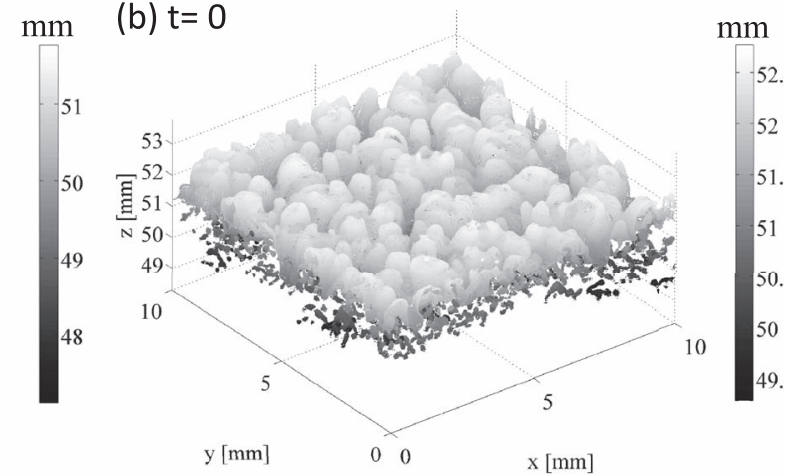

(d) $t=0.35$
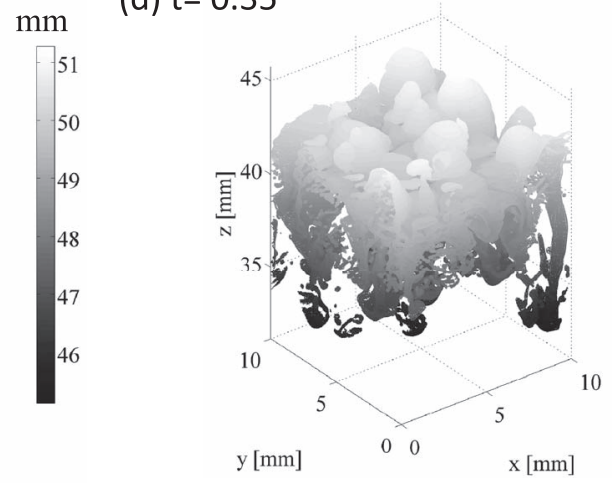

$\mathrm{mm}$

FIG. 6. The interface between the air (bottom) and $\mathrm{SF}_{6}$ (top) in four typical frames, in a multi-mode numerical simulation with $M_{i}=1.2$ for the incident shock wave, $\left\langle\lambda_{0}\right\rangle=0.4 \mathrm{~mm},\left\langle a_{0}^{*}\right\rangle /\left\langle\lambda_{0}\right\rangle=0.2$, and $L=80 \mathrm{~mm} . \mathrm{t}=0$ denotes the time of minimal MZ amplitude. 



FIG. 7. (a) MZ width (dashed-dotted line, black), spike front height (dashed line, red) and bubble front height (solid line, blue) and (b) average, maximal, and minimal wavelength (blue circles, black squares, and red triangles, respectively) as a function of time, obtained in the wide distribution multi-mode numerical simulations with $M_{i}=1.2,\left\langle\lambda_{0}\right\rangle=0.4 \mathrm{~mm}$, and $L=$ $80 \mathrm{~mm} . \mathrm{t}=0$ denotes the time of minimal MZ amplitude.

passage of the reshock. As a result, the average bubble size grows in time, while small bubbles are washed away by large bubbles.

In Fig. 7(a), the heights of the fronts of the bubbles and spikes and the MZ width are presented. It can be seen that the MZ evolves after the passage of the incident shock, reaching an overall width of about $4.5 \mathrm{~mm}$, with bubble to spike fronts amplitudes ratio of about 1/3. After the passage of the reshock, the growth rates increase sharply for both the bubbles and the spikes, and after a short period of having constant velocities (during the phase reversal), the bubble front amplitude continues to grow in a decaying fashion. The average, minimal and maximal bubble wavelengths are presented in Fig. 7(b). The maximal and minimal wavelengths are simply taken as the maximal and minimal wavelengths over the bubble front, respectively, and the average wavelength is extracted using the relation $\langle\lambda\rangle=\sqrt{S / N}$. It can be seen that the wavelength grows throughout the entire simulation, due to bubble merging. However, the rate (i.e., $d\langle\lambda\rangle / d t$ ) increases sharply after the passage of the reshock, as it linearly depends on the bubble front velocity. The average and maximal wavelength growth is by a factor of about 4 , when most of the increase is evident after the reshock, indicating an enhanced bubble merging. Note, that the amplitude to wavelength ratio predicted in the simulation reaches a constant value of $\sim 1.2$ at $t \approx-0.4 \mathrm{~ms}$. This indicates that the merging rate in the simulation reaches a constant value as well.

Fig. 8 presents the bubble front amplitudes of the MZ prior to reshock (Figs. 8(a) and 8(b)) and at the arrival of the rarefaction from the end wall (Figs. 8(b) and 8(c)), for the two initial bubble size distributions used in the multi-mode numerical simulations (i.e., narrow and wide distributions, with $\left\langle\lambda_{0}\right\rangle=0.35$ and $0.4 \mathrm{~mm}$, respectively). Both simulations were done with the same $\left\langle a_{0}^{*}\right\rangle /\left\langle\lambda_{0}\right\rangle$ ratio, of 0.2. It can be seen, that both simulations predict a decrease in the number of bubbles as a result of reshock passage. However, the narrow distribution simulation shows a larger number of bubbles than the wide distribution one. This suggests that the effect of bubble merging in the narrow distribution case is smaller than the one obtained with the wide initial distribution. This result is expected, since the typical merging time depends upon the merging bubble wavelengths ratio, and naturally, this typical ratio is larger in the wide distribution case. The MZ widths and average wavelengths obtained in both simulations are presented in Fig. 9. It can be seen, that the MZ width obtained with the wide distribution is larger than the one obtained with the narrow one, both prior and after the reshock. This is a result of the bubble merging dominance in the wide distribution case, previously discussed. One can see that the average wavelength in the narrow distribution case reaches a lower value than the one obtained in the wide distribution case (Fig. 9(b)). The merging rate in the wide distribution simulation is greater than the rate in the narrow distribution simulation by a factor of about 2 . By that we conclude that the wide distribution simulations are dominated by bubble merging, compared to the narrow distribution ones. As will be discussed further below, this fact impacts the initial condition (i.e., initial average wavelengths and amplitudes) required for obtaining good agreement to the experimental results. 

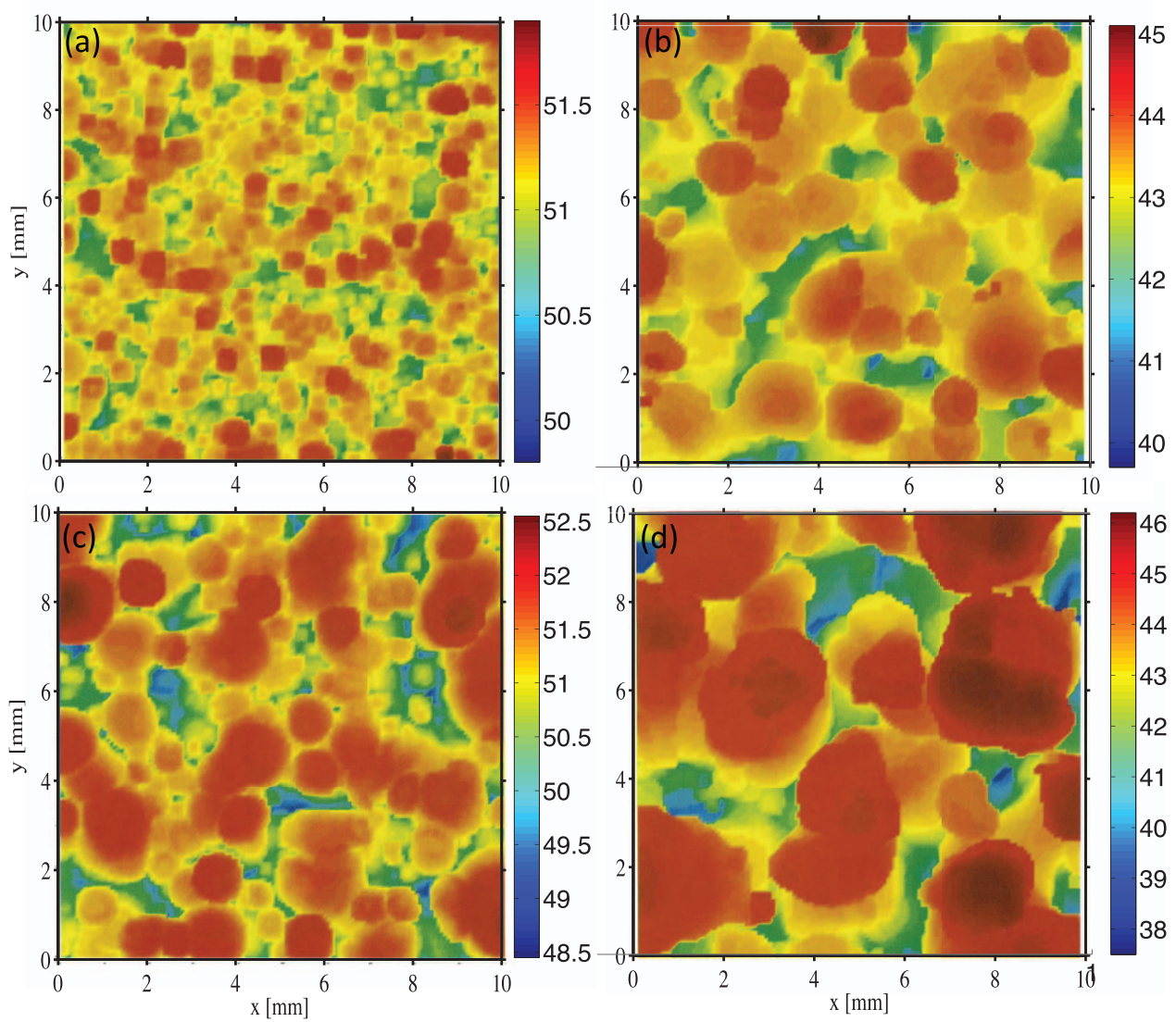

FIG. 8. Bubble fronts obtained from multi-mode numerical simulations done with the narrow (a,b) and wide (c,d) distributions. $M_{i}=1.2$ for the incident shock wave and $L=80 \mathrm{~mm}$. $\left\langle\lambda_{0}\right\rangle=0.35$ and $0.4 \mathrm{~mm}$ for the narrow and wide distributions, respectively. A view from downstream the mixing zone, at $\mathrm{t}=-0.05(\mathrm{a}, \mathrm{c})$ and $\mathrm{t}=0.25(\mathrm{~b}, \mathrm{~d}) \mathrm{ms} . \mathrm{t}=0$ denotes the time of minimal MZ amplitude.

\section{NUMERICAL SIMULATIONS COMPARISON TO EXPERIMENTS AND INITIAL CONDITION DECIPHERMENT}

In this section, the parametric study that was performed in order to resolve the best matching initial conditions of the experiments to be used in the simulations is discussed. We start by comparing
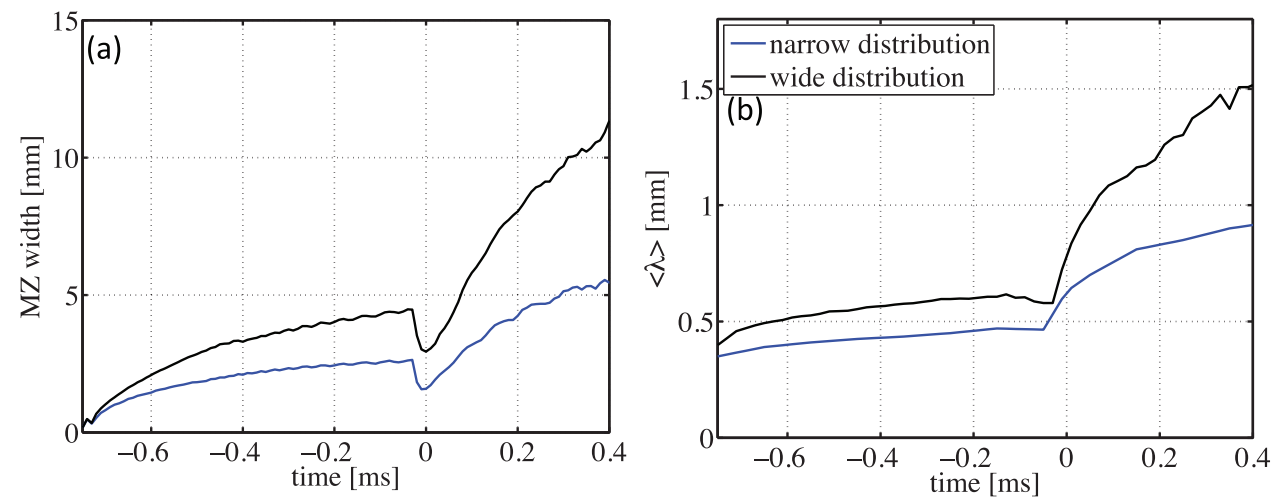

FIG. 9. (a) MZ width and (b) average wavelength as a function of time, obtained in the narrow (blue) and wide (black) distribution multi-mode numerical simulation with $M_{i}=1.2$ and $L=80 \mathrm{~mm} .\left\langle\lambda_{0}\right\rangle=0.35$ and $0.4 \mathrm{~mm}$ for the narrow and wide distributions, respectively. $\mathrm{t}=0$ denotes the time of minimal MZ amplitude. 
TABLE III. Multi-mode narrow distribution numerical simulations summary, $M_{i}=1.2, L=80 \mathrm{~mm}$.

\begin{tabular}{llcc}
\hline \hline $\begin{array}{l}\left\langle\lambda_{0}\right\rangle \\
(\mathrm{mm})\end{array}$ & $\begin{array}{c}\text { Amplitude at reshock arrival } \\
(\mathrm{mm})\end{array}$ & $\begin{array}{c}\text { MZ growth rate after reshock } \\
(\mathrm{mm} / \mathrm{ms})\end{array}$ \\
\hline 0.35 & $\left\langle a_{0}^{*}\right\rangle /\left\langle\lambda_{0}\right\rangle$ & $2.5-5.0$ & $11 \pm 1$ \\
0.5 & $0.2-0.8$ & $2.6-4.9$ & $13.5 \pm 2$ \\
0.7 & $0.13-0.4$ & $2.7-5.0$ & $15.5 \pm 2.5$ \\
1.4 & $0.06-0.32$ & $3.1-5.0$ & $19.5 \pm 2$ \\
2.8 & $0.04-0.11$ & $3.4-5.1$ & $23.1 \pm 1.5$ \\
5.6 & $0.03-0.06$ & $3.2-5.0$ & $23.5 \pm 2$ \\
\hline \hline
\end{tabular}

with the experiments conducted with an end-wall positioned at $80 \mathrm{~mm}$, and then turn to further comparisons, for end-wall positions of 172 and $235 \mathrm{~mm}$, and different incident shock wave Mach numbers.

\section{A. End-wall position at $80 \mathrm{~mm}$}

As discussed in Sec. I B, the first stage of the simulations was carried out with an end-wall position of $80 \mathrm{~mm}$, in order to find the best matching initial wave lengths and amplitudes for each set of initial perturbation (i.e., multi-mode with a wide or a narrow distributions) to the experimental results. The range of initial wavelengths used in the simulations was chosen based on the postexperiment analysis of the membrane fragments, discusses in Sec. I A.

The multi-mode narrow distribution numerical simulation data and results are summarized in Table III and Fig. 10. It can be seen, that good agreement is evident for initial average wavelengths in the range of 1.4-5.6 $\mathrm{mm}$. However, the agreement is achieved over a very narrow range of initial amplitudes. It is also evident that the growth rate after the reshock increase with the increases in the initial average wavelength, and lower initial amplitudes are needed for larger $\left\langle\lambda_{0}\right\rangle$ in order to agree with the experiment. This result is expected, since the terminal velocity of a single bubble is proportional to its wavelength, $u_{b} \sim \lambda t^{-1}$. The growth rate after the reshock is weakly depended upon its amplitude at reshock arrival, as was evident in the experiments of Leinov et al. ${ }^{46}$

The multi-mode wide distribution numerical simulations data and results are summarized in Table IV and Fig. 11. It can be seen, that good agreement is evident for initial average wavelengths in the range of $0.3-0.5 \mathrm{~mm}$, for a relatively large range of initial amplitudes to wavelength ratios $\left(\left\langle a_{0}^{*}\right\rangle /\left\langle\lambda_{0}\right\rangle=0.12-0.33,0.125-0.2\right.$, and $0.09-0.15$ for $\lambda_{0}=0.3,0.4$, and $0.5 \mathrm{~mm}$, respectively). Again, the MZ growth rate after the reshock increases with the initial average wavelength $\left\langle\lambda_{0}\right\rangle$, and lower initial amplitudes are needed for larger $\left\langle\lambda_{0}\right\rangle$ in order to agree with the experiment. Similar to the narrow distribution numerical simulations, the MZ growth rate weakly depends on the MZ width at the reshock arrival for all initial wavelengths simulated, except for the $\left\langle\lambda_{0}\right\rangle=0.2 \mathrm{~mm}$ case. For $\left\langle\lambda_{0}\right\rangle$ greater than $0.2 \mathrm{~mm}$, the variance in the MZ growth rate after the reshock passage is about $10 \%$.

This section is summarized by observing that both initial conditions sets were capable of reproducing the experimental results that were obtained with $M_{i}=1.2$ and $L=80 \mathrm{~mm}$. Good agreement to the experiments was evident for the $\left\langle a_{0}^{*}\right\rangle /\left\langle\lambda_{0}\right\rangle=0.025-0.1$ and $\left\langle\lambda_{0}\right\rangle=1.4-5.6 \mathrm{~mm}$ for the narrow distribution multi-mode simulations $(5.6 \mathrm{~mm}$ is chosen for further comparisons), and $\left\langle a_{0}^{*}\right\rangle /\left\langle\lambda_{0}\right\rangle=0.1-0.25$ and $\left\langle\lambda_{0}\right\rangle=0.3-0.5 \mathrm{~mm}$ for the wide distribution multi-mode simulations.

\section{B. End-wall positions at 172 and $235 \mathrm{~mm}$}

Since the numerical simulations that were performed with both initial condition sets resulted in good agreement with the experiments that were conducted with end-wall position of $80 \mathrm{~mm}$, another set of numerical simulations was performed, in order to check their validity for end-wall positions of 172 and $235 \mathrm{~mm}$. For each set, the numerical simulations were performed with the specific initial conditions that obtained good agreement over a two standard deviations $(2 \sigma)$ range, in order to include the experimental uncertainty of the incident shock wave Mach number. The main difference between the three end-wall positions after the reshock is the arrival time of the rarefaction wave to 

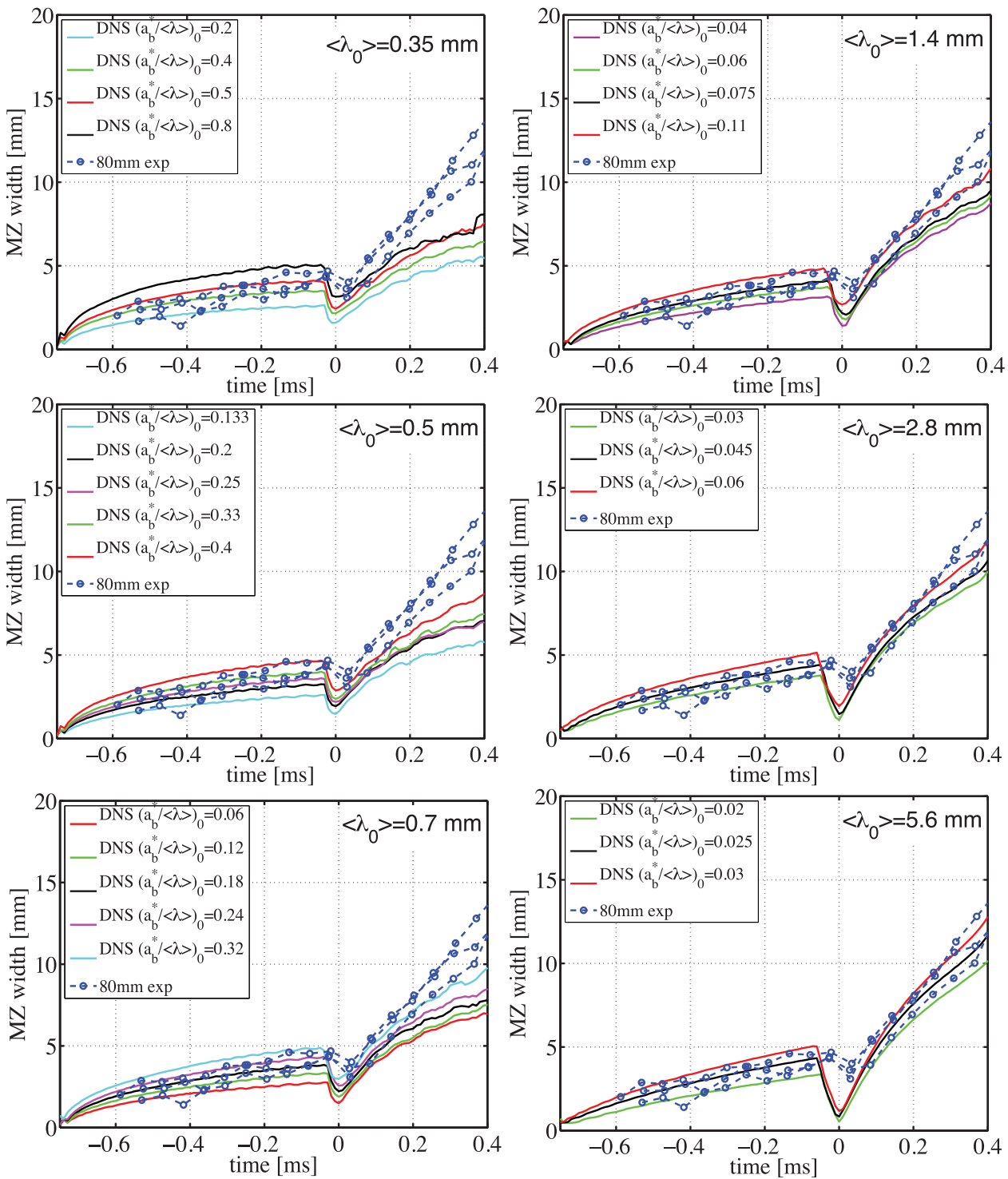

FIG. 10. MZ width as a function of time, obtained in the narrow distribution multi-mode numerical simulations $\left(M_{i}=1.2\right.$, $L=80 \mathrm{~mm}$, smooth lines) and the experiments. ${ }^{46} \mathrm{t}=0$ denotes the time of minimal MZ amplitude.

the MZ (0.4, 0.9, and $1.2 \mathrm{~ms}$ for the $L=80,172$, and $235 \mathrm{~mm}$ cases, respectively). The reshock arrival time was also different in the three cases, $0.78,1.58$, and $2.11 \mathrm{~ms}$ for the $L=80,172$, and $235 \mathrm{~mm}$ cases, respectively.

TABLE IV. Multi-mode wide distribution numerical simulation summary, $M_{i}=1.2, L=80 \mathrm{~mm}$

\begin{tabular}{llcc}
\hline \hline $\begin{array}{l}\left\langle\lambda_{0}\right\rangle \\
(\mathrm{mm})\end{array}$ & \multicolumn{1}{c}{$\begin{array}{c}\text { Amplitude at reshock arrival } \\
(\mathrm{mm})\end{array}$} & $\begin{array}{c}\text { MZ growth rate after reshock } \\
(\mathrm{mm} / \mathrm{ms})\end{array}$ \\
\hline 0.2 & $0.12-0.7$ & $2.6-5.2$ & $13.0 \pm 3.0$ \\
0.3 & $0.12-0.5$ & $3.5-5.4$ & $21.6 \pm 1.5$ \\
0.4 & $0.065-0.72$ & $2.7-8.0$ & $22.3 \pm 1.0$ \\
0.5 & $0.06-0.2$ & $2.7-3.0$ & $24.5 \pm 1.0$ \\
0.6 & $0.03-0.24$ & $2.6-8.0$ & $29.0 \pm 3.0$ \\
0.8 & $0.035-0.375$ & $2.7-9.7$ & $32.3 \pm 2.5$ \\
\hline \hline
\end{tabular}



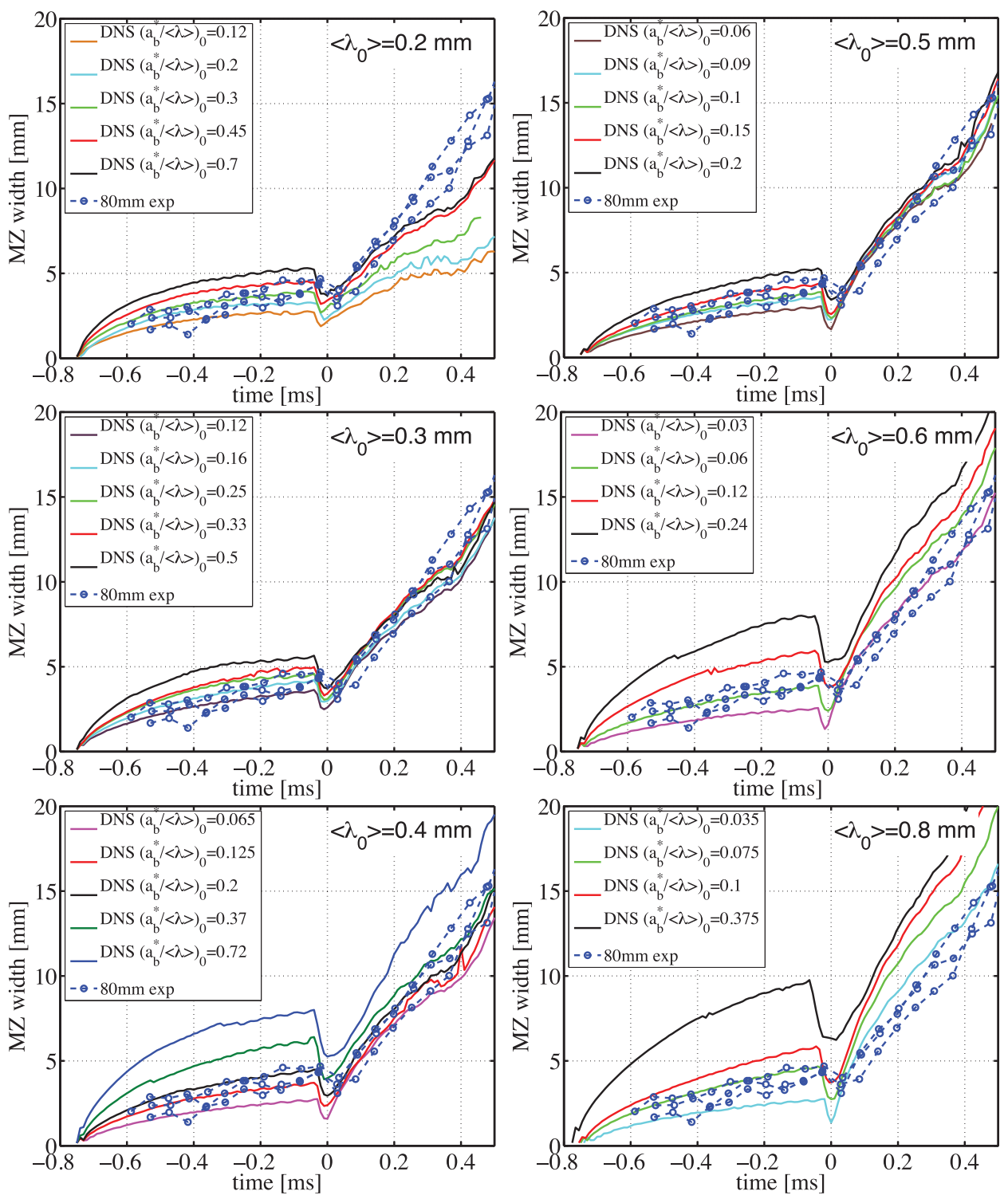

FIG. 11. MZ width as a function of time, obtained in the wide distribution multi-mode numerical simulations $\left(M_{i}=1.2, L\right.$ $=80 \mathrm{~mm}$, smooth lines) and the experiments. ${ }^{46} \mathrm{t}=0$ denotes the time of minimal $\mathrm{MZ}$ amplitude.

The comparison between the numerical simulations and the experimental results is presented in Fig. 12, in reference to the time of the reshock arrival in the $L=235 \mathrm{~mm}$ case. For reference, the results for the $L=80 \mathrm{~mm}$ case are also presented. When examining the agreement between the multi-mode narrow distribution numerical simulations $\left(\left\langle\lambda_{0}\right\rangle=5.6 \mathrm{~mm}\right)$ to the experimental results (Fig. 12(a)), one finds that while the agreement after the passage of the reshock is reasonable, the MZ evolution does not agree with the experiments before the reshock arrival. Therefore, we can rule out the multi-mode, narrow distribution as the possible initial distribution in the experiments. Note that although we present here only the results for $\left\langle\lambda_{0}\right\rangle=5.6 \mathrm{~mm}$, the conclusions are similar for other initial wavelengths which agreed well to the experiments done with $L=80 \mathrm{~mm}$, e.g., $\left\langle\lambda_{0}\right\rangle$ $=1.4 \mathrm{~mm}$.

The comparison to the experiments for the multi-mode wide distribution numerical simulations results $\left(\left\langle\lambda_{0}\right\rangle=0.4 \mathrm{~mm}\right)$ is presented in Fig. 12(b). In contrast to the multi-mode narrow distribution, the wide distribution numerical simulation agrees well with the experiments for the three end-wall positions. The good agreement that is evident allows one to determine that the wide distribution 

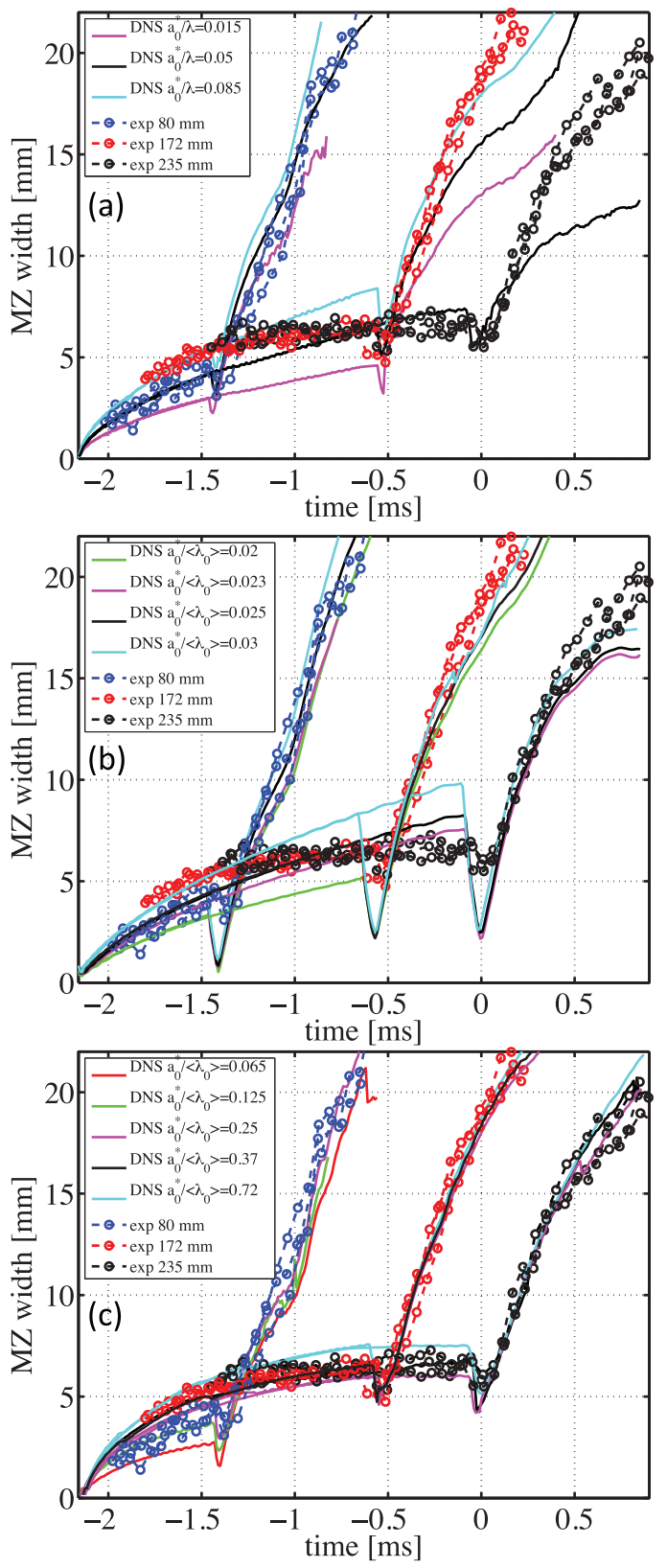

FIG. 12. MZ width as a function of time for $M_{i}=1.2$ and $L=80,172$, and $235 \mathrm{~mm}$ (smooth lines), obtained in the experiments ${ }^{46}$ and in the numerical simulations with, (a) narrow distribution multi-mode with $\left\langle\lambda_{0}\right\rangle=5.6 \mathrm{~mm}$ and (b) wide distribution multi-mode with $\left\langle\lambda_{0}\right\rangle=0.4 \mathrm{~mm} . \mathrm{t}=0$ denotes the minimal $\mathrm{MZ}$ amplitude time in the $235 \mathrm{~mm}$ case.

multi-mode initial condition characterizes the experiments with high confidence. This will now be further validated by comparing the multi-mode wide distribution numerical simulations with the experimental results for different incident shock wave Mach numbers $\left(M_{i}=1.15\right.$ and 1.3).

\section{Different incident shock wave Mach numbers}

After ruling out the multi-mode narrow distribution as characterizing the experimental MZ evolution, a further validation of the multi-mode wide distribution case was carried out. The $\left\langle\lambda_{0}\right\rangle$ $=0.4 \mathrm{~mm}$ numerical simulations are compared to the experiments done with $M_{i}=1.1$ and 1.3 in Fig. 13. The obtained good agreement confirms the ability of the numerical simulations with the multi-mode wide distribution to reproduce the experiments over a wide range of constraints. It 

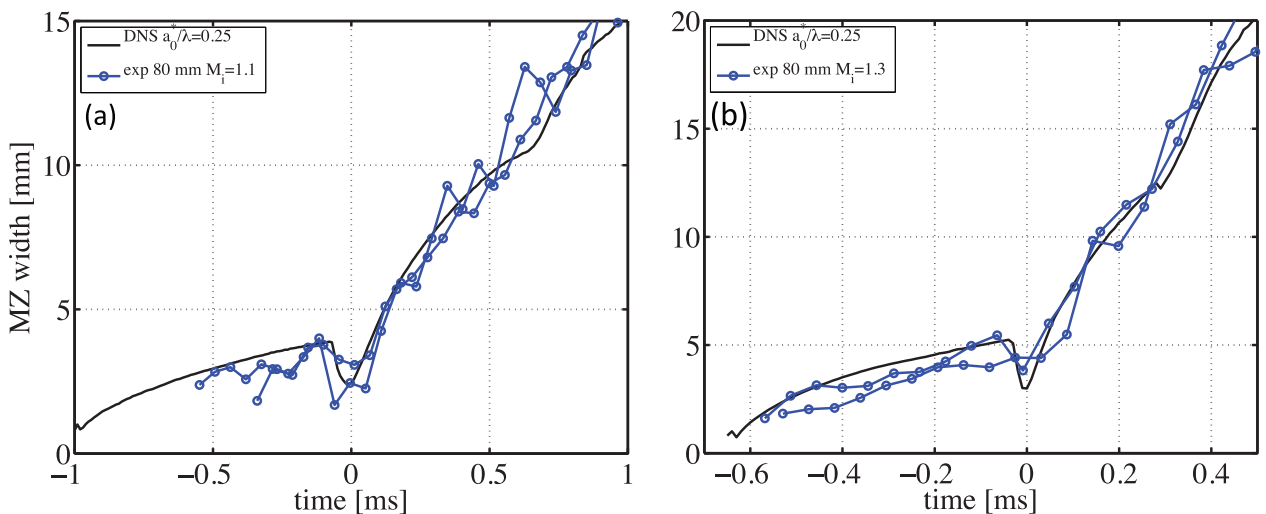

FIG. 13. MZ width as a function of time for $L=80 \mathrm{~mm}$ and (a) $M_{i}=1.1$, (b) $M_{i}=1.3$, obtained in the experiments ${ }^{46}$ (blue) and in the wide distribution multi-mode numerical simulations (black) with $\langle\lambda\rangle=0.4 \mathrm{~mm} . \mathrm{t}=0$ denotes the minimal $\mathrm{MZ}$ amplitude time.

should be noted that the numerical simulation results showed the same dependencies on the post reshock growth rate as the experiments, namely, weak dependency on the MZ amplitude at the reshock arrival and high dependency on the incident shock wave Mach number.

In summary, by concluding that although the two types of initial perturbations could reproduce the experimental results for Case $1\left(L=80 \mathrm{~mm}\right.$, and $M_{i}=1.2$, see Table I), only one type could reproduce the experiments under all the chosen constraints (Cases 2-5 in Table I). This allows to classify with high confidence the experimental initial condition as a multi-mode, wide distribution one, with $\left\langle\lambda_{0}\right\rangle \approx 0.3-0.5 \mathrm{~mm}$.

\section{A SIMPLE MODEL FOR RESHOCKED RM BUBBLE DYNAMICS}

After the analysis by means of the full numerical simulations, we turn to an attempt to better understand the bubble evolution dynamics, by deriving a simple model. Such a model can assist in analyzing the numerical simulation results, and therefore in better understanding the MZ typical flow regime. We now discuss the details of the model, giving a summary of its main features in Table V. It was found earlier that the large scale evolution of the single-mode shock-reshock interaction could be divided into three characteristic time periods:

1. From the incident shock wave passage until the reshock arrival at the bubble front.

2. Phase reversal, during which the bubble velocity is approximately constant and similar to the spike velocity, but lower than the linear velocity.

3. From the end of the phase reversal until the arrival of the rarefaction wave. At this period the bubble velocity quickly decays to the asymptotic solution of $u_{b} \sim \lambda / t$.

These three stages allow the definition of time markers; the time of arrival of the incident shock wave $\left(t_{i}\right)$, the reshock $\left(t_{r}\right)$, and the rarefaction wave $\left(t_{e}\right)$. These time markers can be easily determined using the velocities of the shock and rarefaction waves and the bubble amplitude, which is obtained by the model. Another required time marker is the time at which the phase reversal ends, $\left(t_{p}\right)$, which helps defining the phase reversal time scale $\left(t_{p}-t_{r}\right)$.

TABLE V. Summary of the different expressions, time scales, and initial amplitudes used in the model presented in this table, at each phase of the interaction. The bubble velocity reduction factor, $F=u_{b i} / U_{R M}$ is described in Eq. (5).

\begin{tabular}{lccc}
\hline \hline Initial condition & Before rechock & Phase reversal & After reshock \\
\hline Bubble velocity & $u_{b}=F \cdot U_{R M} \frac{1+B t}{1+D t+E t^{2}}$ & $u_{b}= \pm F \cdot U_{R M}$ & $u_{b}=F \cdot U_{R M} \frac{1+B t}{1+D t+E t^{2}}$ \\
Time period & $t_{r}-t_{i}$ & $t_{p}-t_{r}$ & $t_{e}-t_{p}$ \\
Initial amplitude & $a_{0}^{*}\left(t_{i}\right)$ & $a_{0}^{*}\left(t_{r}\right)$ & $a_{0}^{*}\left(t_{r}\right)$ \\
Relevant equations & $2,4,5$ & 2,5 & $2,4,5$ \\
\hline \hline
\end{tabular}


The bubble velocity, $u_{b}$, for each time period can be obtained using the well-known formulation of Sadot et al., ${ }^{31}$ multiplied by a high amplitude reduction factor, $F$ :

$$
u_{b}=F \cdot U_{R M} \frac{1+B t}{1+D t+E t^{2}}
$$

where $B=F U_{R M} k, D=(1+A) F U_{R M} k$, and $E=F U_{R M}^{2} k^{2}$. It can be seen that the limit $t \rightarrow \infty$, Eq. (4) yields the correct asymptotic velocity of $u_{b} \sim \lambda /(2 \pi t)$. The reduction factor $F=u_{b i} / U_{R M}$ represents the ratio between the actual initial bubble velocity and the velocity obtained using the impulsive model $^{1}$ (Eq. (2)). This formula was validated in past studies against two-dimensional experimental results ${ }^{31,44}$ for the $F=1$, single shock case and was found to be robust. Dimonte et $a l .{ }^{28}$ presented a model including a correction term for high initial amplitudes, again for a single shock, which was validated against numerical and experimental results. The reduction factor in their study was fitted to numerical results, as a function of the initial amplitude to the wavelength ratio.

In the present model, the reduction factor, $F$, is obtained using the model of Rikanati et al. ${ }^{27}$ which analytically gives the initial velocity, $u_{b i}$, as a function of the initial amplitude $a_{0}$, the perturbation wavelength, $\lambda$, and the fluids properties:

$$
\begin{aligned}
u_{b i}= & -\operatorname{Re}\left\{f\left(M, \rho_{1}, \rho_{2}, \gamma_{1}, \gamma_{2}, p_{1}, p_{2}\right) .\right. \\
& \int_{0}^{\lambda} \frac{1}{2 \lambda} \frac{\sin \alpha^{-}(x)}{\cos \alpha^{+}(x)} . \\
& \left.\cot \left[\pi \frac{-x+i f_{p}\left(a_{0}-y(x)\right)}{\lambda}\right] d x\right\},
\end{aligned}
$$

where $\operatorname{alpha}^{+}(x)$ and $\operatorname{alpha}^{-}(x)$ are the pre and post rechock angles between the shock an the interface, respectively, and $f_{p}$ is the shock compression factor, $f_{p}=\left(u_{s}-\Delta_{u}\right) / u_{s}$. Dividing Eq. (5) by Eq. (2) yields the reduction factor, $F$. One should note that in order to use Eq. (5), the bubble should be assumed to be axially symmetric. In addition, it should be assumed that the bubble/spike asymmetry at the reshock arrival could be considered by using $\left.y(x)=a_{0}\left[1-y_{0}\right]\left\{1+\max \left[-\operatorname{sign}\left(y_{0}\right), 0\right)\right]\right\}$ in Eq. (5), where $y_{0}=\cos (2 \pi x / \lambda)$. The velocity reduction factor, $F$, as a function of the bubble amplitude to wavelength ratio is presented in Fig. 14, for a symmetric (dashed line) and asymmetric (solid line) perturbations. It can be seen that $a / \lambda=0.5$ yields $F \approx 1 / 3$ for the asymmetric case, in good agreement with the numerical simulations results presented in Sec. II A (see Fig. 4).

For the first time period $\left(t_{i}<t<t_{r}\right)$ Eq. (4) is simply used with the initial post shock amplitude from the numerical simulations (computed separately). For evaluating the phase reversal time period $\left(t_{r}<t<t_{p}\right)$ its time scale $\left(t_{p}-t_{r}\right)$ should be assessed first. This can be obtained by considering the



FIG. 14. The velocity reduction factor F for a symmetric (dashed line) and asymmetric (solid line) perturbation as derived using Eq. (5). 
absolute value of the bubble velocity at this stage to be constant, and therefore, $t_{p}-t_{r}=\left[h_{b}\left(t_{r}\right)+\right.$ $\left.h_{s}\left(t_{r}\right)\right] /\left|u_{b i}\right|$, since the phase reversal takes place on the overall MZ width. The velocity magnitude at this stage can be determined from Eq. (4), by considering the amplitude that was obtained at the end of the first stage, $h_{b}\left(t_{r}\right)$, as the initial bubble amplitude. Naturally, the obtained bubble velocity will be negative until $t=0$, and positive after that. The MZ width $h_{b}+h_{s}$ was simply estimated by taking the spike amplitude (for $A \sim 0.7$ ) to be about $3 h_{b}$. The bubble velocity during the last time period $\left(t_{p}<t<t_{r}\right)$ was estimated by using Eq. (4) with the appropriate velocity reduction factor, and $t_{p}$ as the initial time. After the bubble velocity was obtained for all the time periods, the bubble height could be derived with a time integral over the velocity.

In order to expand the model for the multi-mode case, bubble merging must be considered. Following Oron et al. ${ }^{26}$ the 3D bubble size distribution $f(\lambda, t)$ was obtained using

$$
\begin{aligned}
& N(t) \frac{\partial f(\lambda, t)}{\partial t}=-2 f(\lambda, t) \int_{0}^{\infty} f\left(\lambda^{\prime}, t\right) \omega\left(\lambda, \lambda^{\prime}\right) d \lambda^{\prime} \\
+ & \int_{0}^{\lambda} f\left(\sqrt{\lambda^{2}-\lambda^{\prime 2}}, t\right) f\left(\lambda^{\prime}, t\right) \omega\left(\sqrt{\lambda^{2}-\lambda^{\prime 2}}, \lambda^{\prime}\right) d \lambda^{\prime},
\end{aligned}
$$

where $N(t)=\int_{0}^{\infty} f(\lambda, t) d \lambda$ is the total number of bubbles at time $t$, and $\omega\left(\lambda, \lambda^{\prime}\right)$ is the merging rate between bubbles having wavelengths $\lambda$ and $\lambda^{\prime}$.

Equation (6) was used to compute the bubble size distribution during the above mentioned time periods while assuming that the merging rate between every two bubbles is governed by the large one, and therefore defining:

$$
\omega\left(\lambda, \lambda^{\prime}\right)=\frac{u_{b}(\lambda)}{\lambda} \bar{\omega}(q)
$$

where $\omega\left(\lambda, \lambda^{\prime}\right)$ in Eq. (7) is the merging rate, $u_{b}(\lambda)$ is obtained for each wavelength using Eq. (4), and $\bar{\omega}(q)$ is the non-dimensional merging rate, ${ }^{26}$ that depends on the wavelength ratio, $q=\lambda / \lambda^{\prime}$, of the merging bubbles. In the present study, $\bar{\omega}$ was computed using the two-dimensional potential flow model of Hecht et al. ${ }^{11}$ as was done by Oron et al. ${ }^{26}$ After the bubble size distribution was obtained throughout the entire process, one can obtain the average wavelength $\langle\lambda\rangle$. The average wavelength of the rising bubbles, $\left\langle\lambda_{u}\right\rangle$ can be obtained by calculating the average wavelength while considering only the wavelengths with a constant or growing number of bubbles. For each size distribution, $f(\lambda, t)$, the bubble front velocity is given by Eq. (4) and using $\left\langle\lambda_{u}\right\rangle$ as the wavelength. Hence, the increasing wavelength effect could be considered in the model.

\section{A. Model comparisons to the numerical simulations results: Single-mode}

In the following, the model predictions compared to numerical simulation results are presented for the single-mode initial perturbation. In order to test the ability of the model to capture the physics

TABLE VI. Single-mode numerical simulations done for model evaluation. Incident shock wave Mach number: 1.2 and 1.3

\begin{tabular}{|c|c|c|c|c|c|}
\hline Case & Configuration & $\begin{array}{c}\lambda \\
(\mathrm{mm})\end{array}$ & $a_{0}^{*} / \lambda$ & $\begin{array}{c}\Delta u_{1} \\
(\mathrm{~mm} / \mathrm{ms})\end{array}$ & $\begin{array}{c}\Delta u_{2} \\
(\mathrm{~mm} / \mathrm{ms})\end{array}$ \\
\hline 1 & & 3 & 0.002 & & \\
\hline 2 & & 3 & 0.016 & & \\
\hline 3 & $\operatorname{air} / S F_{6}$ & 3 & 0.025 & 67.9 & -24.4 \\
\hline 4 & & 3 & 0.033 & & \\
\hline 5 & & 6 & 0.025 & & \\
\hline 6 & $S F_{6} /$ air & 3 & 0.018 & 89.6 & 31.9 \\
\hline
\end{tabular}
for air/SF 6 and $S F_{6} /$ air configurations, respectively. End wall position $L=80 \mathrm{~mm}$. The initial bubble amplitude values given in the table are the compressed ones, calculated using $a_{0}^{*}=f_{p} a_{0}$, where $f_{p}=\left(u_{s}-\Delta u\right) / u_{s}$, and $u_{s}$ is the shock velocity. ${ }^{10}$ 




FIG. 15. Bubble height obtained from Case $1\left(a_{0}^{*} / \lambda=0.002\right)$ numerical simulations (solid line) and model (dashed line).

of the bubble evolution, several simulations were performed, summarized in Table VI. The notations $\Delta u_{1}$ and $\Delta u_{2}$ refer to the contact surface velocity before and after the reshock, respectively.

In order to check the ability of the model to deal with the reshock problem in a simple case, the model prediction is compared first to Case 1 of the numerical simulation results (see Table VI). Since $a_{0}^{*} / \lambda$ in this case is very small, the bubble amplitude remains in the linear regime (and therefore the bubble and spike symmetry is maintained) until the reshock arrival. As a result, both the phase reversal time scale and the high amplitude effect are negligibly small (i.e., $t_{p}-t_{r} \approx 0.01 \mathrm{~ms}, F \approx$ 1). The comparison is presented in Fig. 15. Good agreement is evident, confirming the ability of Eq. (4) to reproduce the bubble evolution for this simple case.

The bubble height as obtained from the numerical simulation of a heavy/light (Case 6, Table VI) single-mode run and the model is presented in Fig. 16. In this case, when the reshock passes through the MZ, no phase reversal occurs. Also presented is the model prediction, when the high amplitude effect is not considered (i.e., $F=1$ ). It can be seen that the model prediction is in good agreement with the numerical simulation. When the high amplitude effect is not considered, the bubble amplitude after the reshock is overestimated, due to the overestimation of the predicted velocity. Note, that the amplitude to wavelength ratio at the reshock arrival reaches a value of 0.4. The good agreement between the model prediction and the numerical simulation after the reshock further validates the use of Eqs. (4) and (5).

The model prediction for Case 3 (Table VI) is compared to the numerical simulation prediction in Fig. 17. Also presented are the predictions obtained by the impulsive model (Eq. (2)), and the

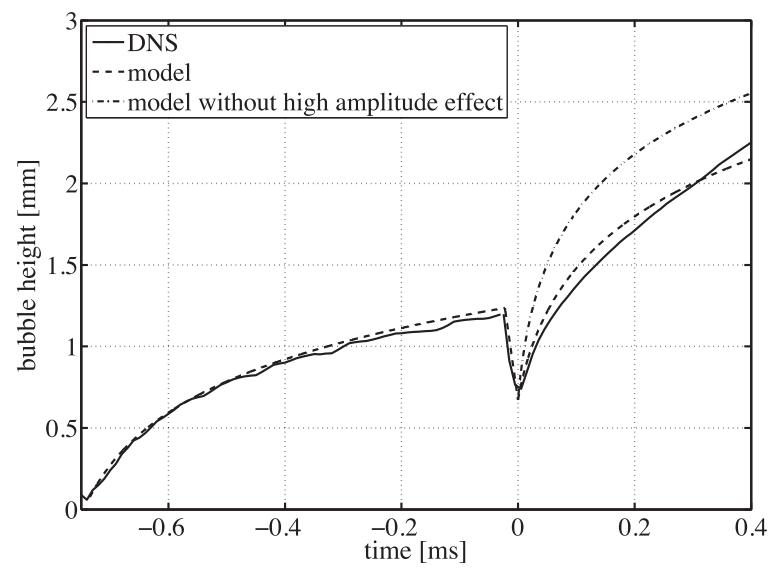

FIG. 16. Bubble height obtained from numerical simulations (solid line) and model (dashed line), $S F_{6}$ - air configuration. The dashed-dotted line corresponds to the model without considering the high amplitude effect (Eq. (5)). 


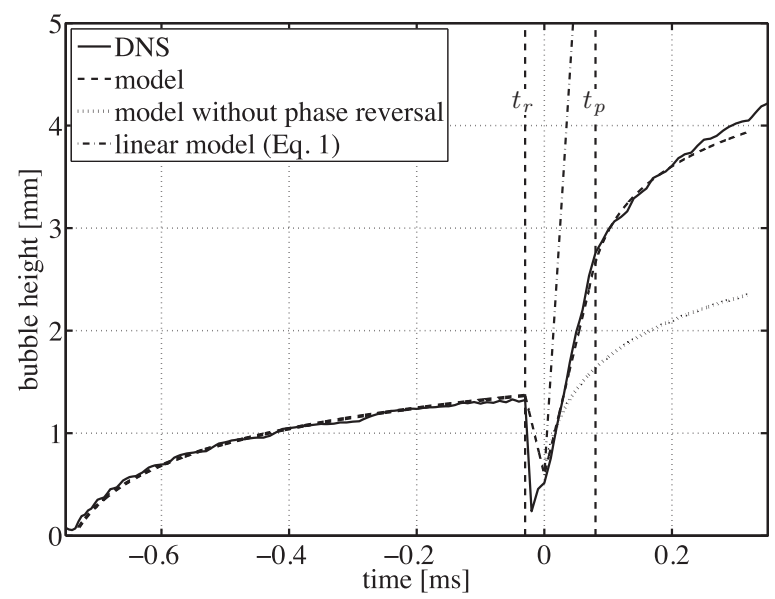

FIG. 17. Bubble height obtained from Case $3\left(a_{0}^{*} / \lambda=0.025\right)$ numerical simulations (solid line) and model with (dashed line) and without (dotted line) phase reversal. Dashed-dotted line represents the linear solution (Eq. (2)). Left and right dashed vertical lines represent the time of reshock arrival $\left(t_{r}\right)$ and the end of phase reversal $\left(t_{p}\right)$, respectively.

present model prediction, when the phase reversal is not considered at $t>0$. It can be seen that the model reproduces well the numerical simulation result, while the linear solution predicts a much larger bubble amplitude. Also, when implementing the model without considering the phase reversal after $t>0$ (dashed line), the predicted bubble amplitude underestimates the numerical simulations result. This result confirms that the use of Eq. (4), combined with the phase reversal estimation and the high amplitude effect captures the physics of bubble evolution. The fact that good agreement is achieved only when the phase reversal is considered, demonstrates its necessity and importance in the model. The predicted growth rate following the phase reversal (marked with the right vertical dashed line) is very similar to the growth rate obtained by the model without considering the phase reversal. This result is expected, since in both cases the model used $t_{r}$ (marked with the left vertical line) as a time mark, and $h_{b}\left(t_{r}\right)$ as an initial amplitude.

In order to examine the initial amplitude effect, the bubble height, as obtained from the model and the numerical simulations are presented in Fig. 18 for $\lambda=3 \mathrm{~mm}$ and initial amplitude to wavelength ratios of $0.016,0.025$, and 0.033 (Cases 2-4 in Table VI). It can be seen that the model well reproduces the numerical simulation results. As expected, the different initial amplitudes yield differences in the bubble heights of about $0.1-0.3 \mathrm{~mm}$ at the reshock arrival. The predicted post reshock growth rates (during the phase reversal) in the model and the numerical simulation are very similar for all the initial amplitudes. This results in two competing effects. Since the pre-reshock

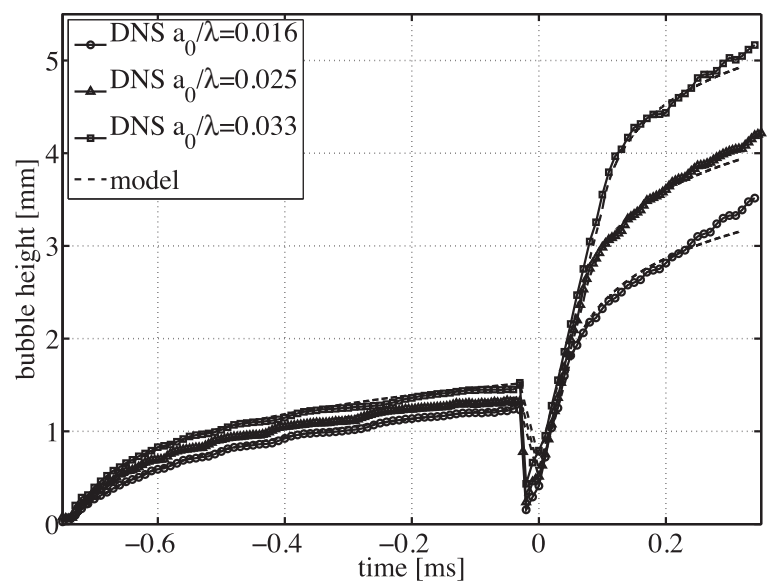

FIG. 18. Bubble height obtained from the $\lambda=3$ (Cases 2-4) $\mathrm{mm}$ and $a_{0}^{*} / \lambda=0.016$ (circles), 0.025 (triangles), and 0.033 (squares) numerical simulations and model (dashed corresponding lines). 


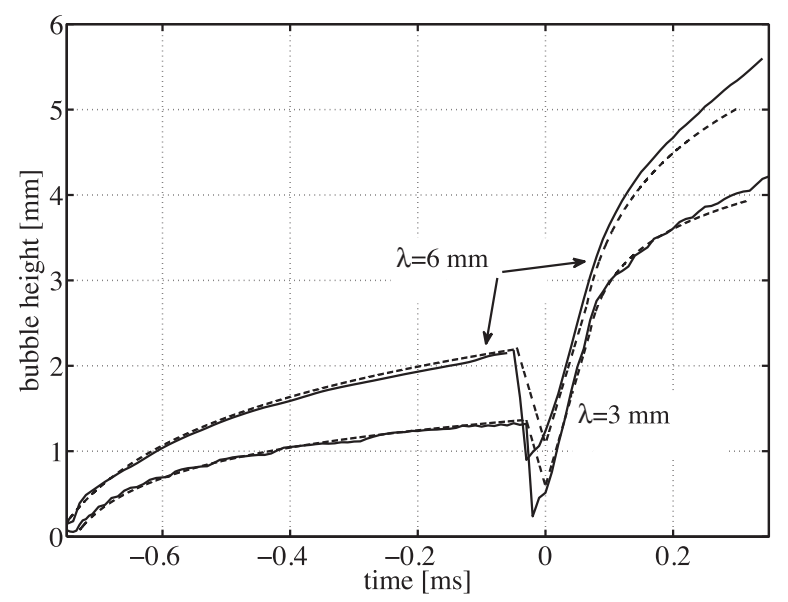

FIG. 19. Bubble height obtained from the $\lambda=3 \mathrm{~mm}$ and $6 \mathrm{~mm}, a_{0}^{*} / \lambda=0.025$ numerical simulations (solid line) and model (dashed corresponding lines).

bubble amplitude grows with initial amplitude, the linear velocity, $U_{R M}$, also grows. However, the velocity reduction factor due to the amplitude at the reshock arrival grows as well, and therefore reduces the initial bubble velocity. These two effects partially compensate for each other, resulting in similar growth rates during the phase reversal. The main difference between the presented three cases is the phase reversal time scale, which grows with the amplitude (i.e., $t_{p}-t_{r} \propto h_{b}+h_{s}$ ). After the phase reversal is completed, the bubble growth rate decays quickly for all initial amplitudes, and reaches the asymptotic regime. Since all simulations were performed with the same wavelength, the bubble growth rates are very similar for all the amplitudes.

In Fig. 19, the bubble height as obtained from the numerical simulation and the model is plotted against time for the $\lambda=3$ and $6 \mathrm{~mm}$ numerical simulations and the model, with $a_{0}^{*} / \lambda=0.025$ (Cases 3 and 5 in Table VI). The agreement between the numerical simulation and the model is good. Before the reshock, the bubble growth rate depends on the wavelength and the amplitude, reaching a higher amplitude in the $\lambda=6 \mathrm{~mm}$ case. After the reshock and during the phase reversal, the bubble growth rate is similar for both wavelengths. This can be expected, since the ratio between the two bubble velocities is $\left(\lambda_{2} / \lambda_{1}\right)\left(a_{1} / a_{2}\right)\left(F_{1} / F_{2}\right)=(6 / 3)(1.2 / 2.1)(0.37 / 0.41) \approx 1$. However, due to the difference in the MZ width at the reshock arrival, the phase reversal time is longer for the $\lambda=$ $6 \mathrm{~mm}$ case, and the bubble amplitude at the end of the phase reversal is larger. After the phase reversal, the $\lambda=6 \mathrm{~mm}$ bubble grows faster than the $\lambda=3 \mathrm{~mm}$ bubble, since the bubble growth rate increases with wavelength, as expected for the asymptotic regime.

\section{B. Model comparisons to the numerical simulation results: Multi-mode}

The normalized bubble size distributions obtained by the model and the multi-mode numerical simulation that obtained the best agreement with the experiments (wide spectrum, $\left\langle a_{0}^{*}\right\rangle /\left\langle\lambda_{0}\right\rangle=0.2$, $\left.\left\langle\lambda_{0}\right\rangle=0.4\right)$ are presented in Fig. 20. The non-dimensional distribution was calculated using $f(\xi)=$ $[\langle\lambda\rangle / N] f(\lambda)$ and $\xi=\lambda /\langle\lambda\rangle$. The asymptotic self-similar size distribution that was predicted by the model is also presented for each sub-figure. The bubble size distribution obtained in the numerical simulation converges towards the self-similar distribution upon the reshock arrival (Figs. 20(a)20(d)), in good agreement with the model predictions. After the passage of the reshock the distribution is diverted from the self-similar one due to a sudden rise in the bubbles velocities (Fig. 20(e)), and then converges to self-similarity (Fig. 20(f)). This result confirms again the dominancy of the inverse cascade process in the wide distribution simulation after the reahock passage. Considering this and following the methodology described in Sec. I B, the good agreement with the experimental results that was obtained only with the wide distribution, implies that the inverse cascade of the bubble merging in the experiments increases due to the passage of the reshock, dominating the flow. By examining the normalized bubble size distribution that was obtained in the numerical simulation with $L=235 \mathrm{~mm}$ (Fig. 21) and comparing it to the asymptotic distribution obtained by the model, 

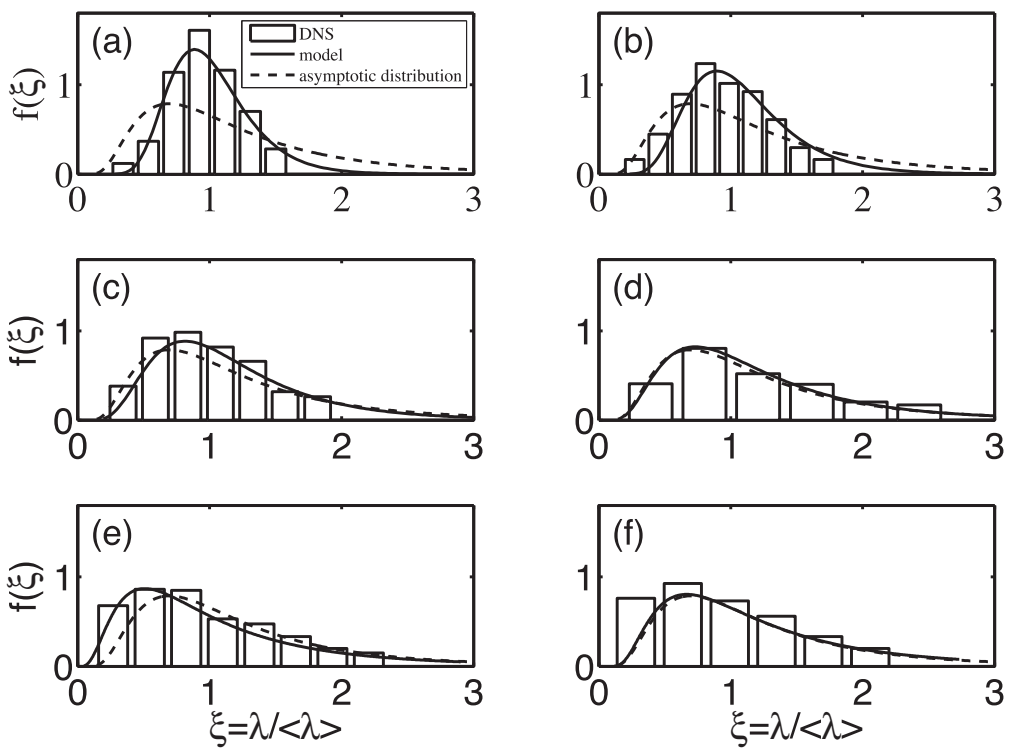

FIG. 20. Normalized bubble size distribution obtained by the numerical simulation (bars) and model (solid line). Dashed line: the asymptotic size distribution obtained by the model. (a) $t=-0.75$ (initial condition), (b) $t=-0.6$, (c) $t=-0.3$, (d) $\mathrm{t}=-0.05$ (reshock arrival to bubble front), (e) $\mathrm{t}=0.1$, and (f) $\mathrm{t}=0.3 . M_{i}=1.2, L=80 \mathrm{~mm}$, and $\left\langle\lambda_{0}\right\rangle=0.4 \mathrm{~mm}$.

one can conclude that the bubble size distribution converge towards self-similarity in the range of the end-wall positions that were experimentally investigated by Leinov et al. ${ }^{46}$

In order to demonstrate the convergence to self-similarity in the model and the numerical simulation, the bubble front power law coefficient, $\theta_{b}$, was extracted from the numerical simulation
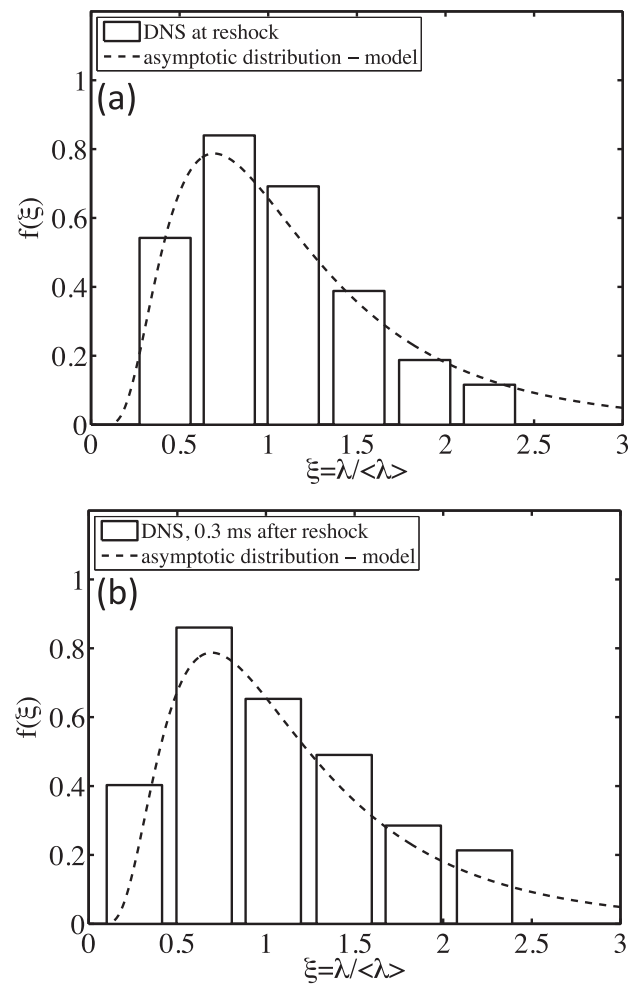

FIG. 21. Normalized bubble size distribution obtained by the numerical simulations (bars) and the asymptotic size distribution obtained by the model (dashed line). (a) $\mathrm{t}=-0.05 \mathrm{~ms}$ (at reshock arrival), (b) $\mathrm{t}=0.3 \mathrm{~ms}$ after the passage of the reshock. $M_{i}=1.2, L=235 \mathrm{~mm}$, and $\left\langle\lambda_{0}\right\rangle=0.4 \mathrm{~mm}$. 

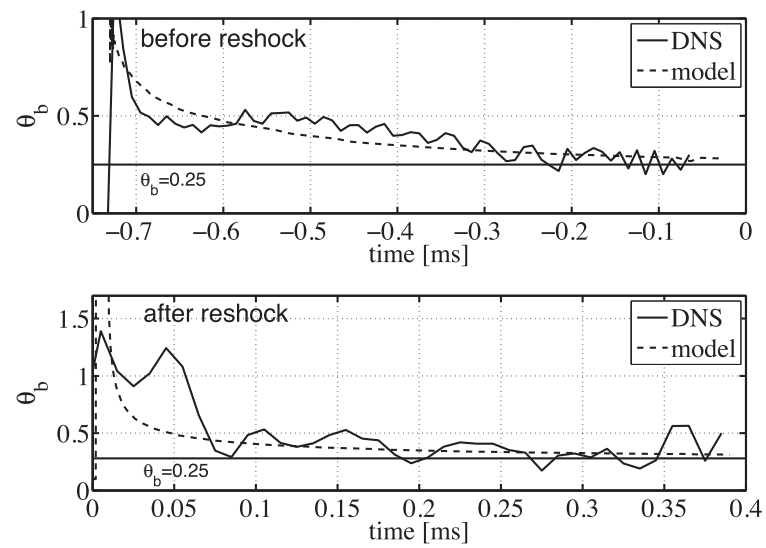

FIG. 22. Power law coefficient $\theta_{b}$ obtained by the numerical simulations (solid line) and model (dashed line) before (upper frame) and after (lower frame) reshock passage. The horizontal solid line represents the asymptotic coefficient obtained by the model. $M_{i}=1.2, L=80 \mathrm{~mm}$, and $\left\langle\lambda_{0}\right\rangle=0.4 \mathrm{~mm}$.
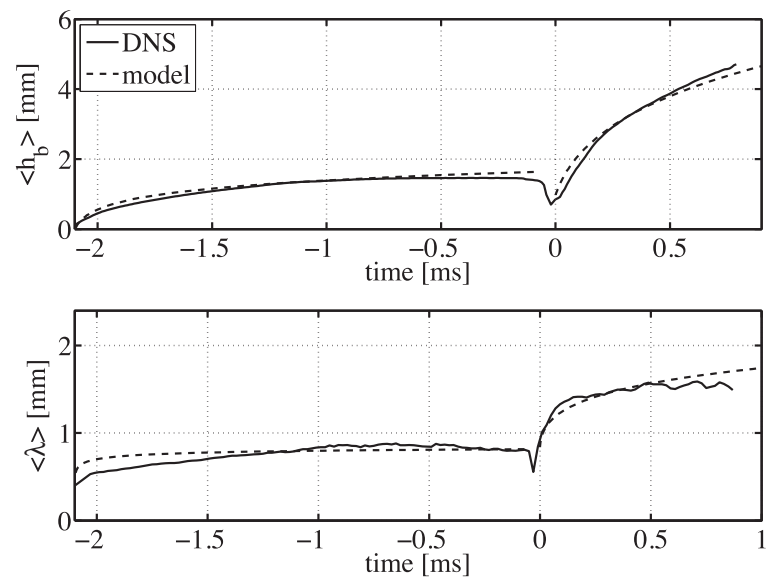

FIG. 23. Bubble front amplitude $\left\langle h_{b}\right\rangle$ (upper frame) and average wavelength $\langle\lambda\rangle$ (lower frame) obtained by the numerical simulations (solid line) and model (dashed line), for $\left\langle\lambda_{0}\right\rangle=0.4 \mathrm{~mm}, M_{i}=1.2$, and $L=235 \mathrm{~mm}$.


FIG. 24. Bubble front amplitude $\left\langle h_{b}\right\rangle$ (upper frame) and average wavelength $\langle\lambda\rangle$ (lower frame) obtained by the numerical simulations (solid line) and model (dashed line), for $M_{i}=1.15$ and 1.3. $L=80 \mathrm{~mm}$ and $\left\langle\lambda_{0}\right\rangle=0.4 \mathrm{~mm}$. 
and the model results, using the relation $\theta_{b}=d\left(\log \left\langle h_{b}\right\rangle\right) / d(\log t)$. The comparison is presented in Fig. 22. Good agreement is evident, and it can be seen that the bubble front converges to the asymptotic value of $\theta_{b}=0.25$ before the reshock arrival (upper frame), as predicted by Oron et al. ${ }^{26}$ The value of $\theta_{b}$ increases due to the passage of the reshock (bottom frame), but converges again to the self-similar asymptotic value at $t \approx 0.1 \mathrm{~ms}$.

To further establish the model validity, the model prediction was compared to the numerical simulation results $\left(\left\langle\lambda_{0}\right\rangle=0.4 \mathrm{~mm}\right)$ in the runs with the different end-wall positions $\left(M_{i}=1.2, L=\right.$ $235 \mathrm{~mm}$, Fig. 23) and the incident shock wave Mach numbers $\left(M_{i}=1.15,1.3, L=80 \mathrm{~mm}\right.$, Fig. 24). For each run, the bubble front height and the average wavelength were compared. It can be seen that the model agrees well with the numerical simulation results. The growth rate, both prior and post the passage of the reshock strongly depends on the incident shock wave Mach number. This result is consistent with the experimental results presented for the overall MZ width in Leinov et al. ${ }^{46}$

\section{v. CONCLUSIONS}

In the present study, the reshock experiments of Leinov et al. ${ }^{46}$ were analysed using a full 3D numerical simulation and a simple model. The study was mainly performed for the light to heavy configuration $\left(a i r / S F_{6}\right)$, for low Mach numbers $(M<1.5)$ and fairly high Atwood numbers $(A \approx 0.7)$.

The study examined the initial perturbation distribution effect on the agreement of the numerical simulation to the experimental results, and by that tried to determine the most possible initial perturbation that initiated the MZ evolution in the experiments. The numerical simulations were compared to the experimental results for three end-wall positions, and three incident shock wave Mach numbers. It was found that the numerical simulation could not reproduce the experiments if the initial perturbation was chosen to be multi-mode with a narrow distribution. It was found that good agreement with the experiments for all end-wall positions and shock Mach numbers could be achieved only when the initial perturbation was chosen to be a multi-mode one with a wide enough bubble size distribution.

A three-dimensional model was presented, and was validated against the simulation results, with good agreement. The model was developed using the formulation of Sadot $e t$ al. ${ }^{31}$ for the single bubble velocity, the model of Rikanati et $a l^{27}$ for high initial amplitudes, and the bubble merger model of Oron $e t$ al. $^{26}$ for the case of a multi-mode random initial perturbation. The model predictions were compared to the results of the numerical simulations results for different initial wavelengths and amplitudes, and also for a single-mode, heavy to light $\left(S F_{6}-\right.$ air $)$ configuration. Good agreement was evident for all cases, confirming the above mentioned analysis.

In the wide distribution multi-mode random perturbation case, it was found that the bubble front evolution was governed by the bubble competition dynamics, both before and after the passage of the reshock. The bubble front characteristics (amplitude, average wavelength, and non-dimensional size distribution) were extracted from the numerical simulation results, and were compared to the model predictions for several cases (different end-wall positions and different incident shock wave Mach numbers, which in turn results in different reshock Mach numbers) with very good agreement. The non-dimensional size distribution of the bubble front approached self-similarity before the reshock arrival, and converged to self-similarity a short time after the reshock passage. The power law coefficient, $\theta_{b}$, was found to converge to about 0.25 both before and after the reshock, with agreement to previous studies. ${ }^{26}$ It is concluded that the use of the bubble merger model of Oron et al. ${ }^{26}$ along with the formulation of Sadot et al. ${ }^{31}$ while considering high amplitude effects ${ }^{27}$ captured the physics of the front evolution both before and after the passage of the reshock.

The good agreement with the experiments that was obtained by the wide bubble size distribution numerical simulations on one hand, and the lack of agreement for the multi-mode, narrow bubble size distribution numerical simulation on the other hand enables one to rule out the possibility of a singlebubble dominated evolution in the experiments. This conclusion is enhanced by the comparisons to the presented model, which confirmed the bubble merging dominance in the simulations done with the wide distribution initial perturbation. 
${ }^{1}$ R. D. Richtmyer, "Taylor instability in shock acceleration of compressible fluids," Commun. Pure Appl. Math. 13, 297-319 (1960).

${ }^{2}$ E. Meshkov, "Instability of the interface of two gases accelerated by a shock wave," Fluid Dyn. 4, 101-104 (1969).

${ }^{3}$ S. W. Haan, S. M. Pollaine, J. D. Lindl, L. J. Suter, R. L. Berger, L. V. Powers, W. E. Alley, P. A. Amendt, J. A. Futterman, W. K. Levedahl, et al., "Design and modeling of ignition targets for the national ignition facility," Phys. Plasmas 2, 2480 (1995).

${ }^{4}$ Y. Aglitskiy, A. Velikovich, M. Karasik, N. Metzler, S. Zalesak, A. Schmitt, L. Phillips, J. Gardner, V. Serlin, J. Weaver, et al., "Basic hydrodynamics of Richtmyer-Meshkov-type growth and oscillations in the inertial confinement fusionrelevant conditions," Philos. Trans. R. Soc., A 368, 1739-1768 (2010).

${ }^{5}$ D. Arnett, B. Fryxell, and E. Mueller, "Instabilities and nonradial motion in SN 1987A," Astrophys. J. 341, L63-L66 (1989).

${ }^{6}$ B. Remington, J. Kane, R. Drake, S. Glendinning, K. Estabrook, R. London, J. Castor, R. Wallace, D. Arnett, E. Liang, et al., "Supernova hydrodynamics experiments on the nova laser," Phys. Plasmas 4, 1994 (1997).

${ }^{7}$ K. Kifonidis, T. Plewa, H.-T. Janka, and E. Müller, "Non-spherical core collapse supernovae," Astron. Astrophys. 408, 621-649 (2003).

${ }^{8}$ C. Kuranz, H.-S. Park, B. Remington, R. Drake, A. Miles, H. Robey, J. Kilkenny, C. Keane, D. Kalantar, C. Huntington, et al., "Astrophysically relevant radiation hydrodynamics experiment at the national ignition facility," Astrophys. Space Sci. 336, 207-211 (2011).

${ }^{9}$ D. Layzer, "On the instability of superposed fluids in a gravitational field," Astrophys. J. 122, 1 (1955).

${ }^{10} \mathrm{~K}$. Meyer and P. Blewett, "Numerical investigation of the stability of a shock-accelerated interface between two fluids," Phys. Fluids 15, 753-759 (1972).

${ }^{11}$ J. Hecht, U. Alon, and D. Shvarts, "Potential flow models of Rayleigh-Taylor and Richtmyer-Meshkov bubble fronts," Phys. Fluids 6, 4019 (1994).

${ }^{12}$ Y. Yang, Q. Zhang, and D. H. Sharp, "Small amplitude theory of Richtmyer-Meshkov instability," Phys. Fluids 6, 18561873 (1994).

${ }^{13}$ D. L. Youngs, "Numerical simulation of mixing by Rayleigh-Taylor and Richtmyer-Meshkov instabilities," Laser Part. Beams 12, 725-750 (1994).

${ }^{14}$ U. Alon, J. Hecht, D. Mukamel, and D. Shvarts, "Scale invariant mixing rates of hydrodynamically unstable interfaces," Phys. Rev. Lett. 72, 2867-2870 (1994).

${ }^{15}$ U. Alon, J. Hecht, D. Ofer, and D. Shvarts, "Power laws and similarity of Rayleigh-Taylor and Richtmyer-Meshkov mixing fronts at all density ratios," Phys. Rev. Lett. 74, 534-537 (1995).

${ }^{16}$ A. L. Velikovich and G. Dimonte, "Nonlinear perturbation theory of the incompressible Richtmyer-Meshkov instability," Phys. Rev. Lett. 76, 3112-3115 (1996).

${ }^{17}$ Q. Zhang and S.-I. Sohn, "An analytical nonlinear theory of Richtmyer-Meshkov instability," Phys. Lett. A 212, 149-155 (1996).

${ }^{18}$ Q. Zhang and S.-I. Sohn, "Nonlinear theory of unstable fluid mixing driven by shock wave," Phys. Fluids 9, 1106-1124 (1997).

${ }^{19}$ A. Rikanati, U. Alon, and D. Shvarts, "Vortex model for the nonlinear evolution of the multimode Richtmyer-Meshkov instability at low Atwood numbers," Phys. Rev. E 58, 7410 (1998).

${ }^{20}$ C. Mügler and S. Gauthier, "Numerical simulations of single-mode Richtmyer-Meshkov experiments," Phys. Rev. E 58, 4548 (1998)

${ }^{21}$ M. Vandenboomgaerde, C. Mügler, and S. Gauthier, “Impulsive model for the Richtmyer-Meshkov instability,” Phys. Rev. E 58, 1874 (1998)

${ }^{22}$ K. O. Mikaelian, "Turbulent mixing generated by Rayleigh-Taylor and Richtmyer-Meshkov instabilities," Physica D 36, 343-357 (1989).

${ }^{23}$ K. O. Mikaelian, "Analytic approach to nonlinear Rayleigh-Taylor and Richtmyer-Meshkov instabilities," Phys. Rev. Lett. 80, 508-511 (1998).

${ }^{24}$ K. O. Mikaelian, "Explicit expressions for the evolution of single-mode Rayleigh-Taylor and Richtmyer-Meshkov instabilities at arbitrary Atwood numbers," Phys. Rev. E 67, 026319 (2003).

${ }^{25}$ G. Dimonte and M. Schneider, "Density ratio dependence of Rayleigh-Taylor mixing for sustained and impulsive acceleration histories," Phys. Fluids 12, 304 (2000).

${ }^{26}$ D. Oron, L. Arazi, D. Kartoon, A. Rikanati, U. Alon, and D. Shvarts, "Dimensionality dependence of the Rayleigh-Taylor and Richtmyer-Meshkov instability late-time scaling laws," Phys. Plasmas 8, 2883-2889 (2001).

${ }^{27}$ A. Rikanati, D. Oron, O. Sadot, and D. Shvarts, "High initial amplitude and high mach number effects on the evolution of the single-mode Richtmyer-Meshkov instability,” Phys. Rev. E 67, 026307 (2003).

${ }^{28}$ G. Dimonte and P. Ramaprabhu, "Simulations and model of the nonlinear Richtmyer-Meshkov instability," Phys. Fluids 22, 014104 (2010)

${ }^{29}$ J. A. McFarland, J. A. Greenough, and D. Ranjan, "Computational parametric study of a Richtmyer-Meshkov instability for an inclined interface," Phys. Rev. E 84, 026303 (2011).

${ }^{30}$ A. Aleshin, E. Lazareva, S. Zaitsev, V. Rozanov, E. Gamalii, and I. Lebo, "Linear, nonlinear, and transient stages in the development of the Richtmyer-Meshkov instability,” Sov. Phys.-Dokl. 35, 159 (1990)

${ }^{31}$ O. Sadot, L. Erez, U. Alon, D. Oron, L. Levin, G. Erez, G. Ben-Dor, and D. Shvarts, "Study of nonlinear evolution of single-mode and two-bubble interaction under Richtmyer-Meshkov instability," Phys. Rev. Lett. 80, 1654-1657 (1998).

${ }^{32}$ C. Niederhaus and J. W. Jacobs, "Experimental study of the Richtmyer-Meshkov instability of incompressible fluids," J. Fluid Mech. 485, 243-277 (2003).

${ }^{33}$ J. Jacobs and V. Krivets, "Experiments on the late-time development of single-mode Richtmyer-Meshkov instability," Phys. Fluids 17, 034105 (2005) 
${ }^{34}$ O. Sadot, "Experimental study of instability of shock-accelerated interface between two media," Ph.D. thesis (Ben-Gurion University, 1998).

${ }^{35}$ O. Sadot, K. Levy, A. Yosef-Hai, D. Cartoon, Y. Elbaz, Y. Srebro, G. Ben-Dor, and D. Shvarts, "Studying hydrodynamic instability using shock-tube experiments," in High Energy Density Laboratory Astrophysics (2005), pp. 305-312.

${ }^{36}$ G. Malamud, C. Di Stefano, Y. Elbaz, C. Huntington, C. Kuranz, P. Keiter, and R. Drake, "A design of a two-dimensional, multimode RM experiment on omega-ep,” High Energy Density Phys. 9, 122-131 (2013).

${ }^{37}$ J. McFarland, D. Reilly, S. Creel, C. McDonald, T. Finn, and D. Ranjan, "Experimental investigation of the inclined interface Richtmyer-Meshkov instability before and after reshock," Exp. Fluids 55, 1640 (2013).

${ }^{38}$ M. Vetter and B. Sturtevant, "Experiments on the Richtmyer-Meshkov instability of an air/SF6 interface," Shock Waves 4, 247-252 (1995).

${ }^{39}$ V. Andronov, S. Bakhrakh, E. Meshkov, V. Mokhov, V. Nikiforov, A. Pevnitskii, and A. Tolshmyakov, "Turbulent mixing at contact surface accelerated by shock waves," Sov. J. Exp. Theor. Phys. 44, 424 (1976).

${ }^{40}$ M. Brouillette and B. Sturtevant, "Richtmyer-Meshkov instability at a continuous interface," AIP Conf. Proc. 208, 284 (1990).

${ }^{41}$ M. Brouillette and B. Sturtevant, "Experiments on the Richtmyer-Meshkov instability: Small-scale perturbations on a plane interface," Phys. Fluids A 5, 916-930 (1993).

${ }^{42}$ L. Houas and I. Chemouni, "Experimental investigation of Richtmyer-Meshkov instability in shock tube," Phys. Fluids 8, 614 (1996).

${ }^{43}$ O. Sadot, L. Erez, D. Oron, G. Erez, G. Ben-Dor, U. Alon, L. Levin, and D. Shvarts, "Studies on the nonlinear evolution of the Richtmyer-Meshkov instability," Astrophys. J., Suppl. Ser. 127, 469 (2000).

${ }^{44}$ B. Collins and J. Jacobs, "PLIF flow visualization and measurements of the Richtmyer-Meshkov instability of an air/SF6 interface," J. Fluid Mech. 464, 113-136 (2002).

${ }^{45}$ E. Leinov, O. Sadot, A. Formoza, G. Malamud, Y. Elbaz, L. Levin, G. Ben-Dor, and D. Shvarts, "Investigation of the Richtmyer-Meshkov instability under re-shock conditions," Phys. Scr. 2008, 014014 (2008).

${ }^{46}$ E. Leinov, G. Malamud, Y. Elbaz, L. Levin, G. Ben-Dor, D. Shvarts, and O. Sadot, "Experimental and numerical investigation of the Richtmyer-Meshkov instability under re-shock conditions," J. Fluid Mech. 626, 449 (2009).

${ }^{47}$ B. Thornber, D. Drikakis, D. Youngs, and R. Williams, "Growth of a Richtmyer-Meshkov turbulent layer after reshock," Phys. Fluids 23, 095107 (2011).

${ }^{48}$ A. Charakhch'yan, "Reshocking at the non-linear stage of Richtmyer-Meshkov instability," Plasma Phys. Controlled Fusion 43, 1169 (2001).

${ }^{49}$ K. O. Mikaelian, "Extended model for Richtmyer-Meshkov mix," Physica D 240, 935-942 (2011).

${ }^{50} \mathrm{O}$. Schilling and M. Latini, "High-order WENO simulations of three-dimensional reshocked Richtmyer-Meshkov instability to late times: dynamics, dependence on initial conditions, and comparisons to experimental data," Acta Math. Sci. 30, 595-620 (2010).

${ }^{51}$ M. Hahn, D. Drikakis, D. Youngs, and R. Williams, "Richtmyer-Meshkov turbulent mixing arising from an inclined material interface with realistic surface perturbations and reshocked flow," Phys. Fluids 23, 046101 (2011).

${ }^{52}$ B. Thornber, D. Drikakis, D. Youngs, and R. Williams, "The influence of initial conditions on turbulent mixing due to Richtmyer-Meshkov instability,” J. Fluid Mech. 654, 99 (2010).

${ }^{53}$ S. Ukai, K. Balakrishnan, and S. Menon, "Growth rate predictions of single-and multi-mode Richtmyer-Meshkov instability with reshock," Shock Waves 21, 533-546 (2011).

${ }^{54}$ D. Shvarts, U. Alon, D. Ofer, R. McCrory, and C. Verdon, "Nonlinear evolution of multimode Rayleigh-Taylor instability in two and three dimensions," Phys. Plasmas 2, 2465 (1995).

${ }^{55}$ J. Hecht, D. Ofer, U. Alon, D. Shvarts, S. Orszag, and R. McCrory, "Three-dimensional simulations and analysis of the nonlinear stage of the Rayleigh-Taylor instability," Laser Particle Beams 13, $423-440$ (1995).

${ }^{56}$ G. Malamud, D. Levi-Hevroni, and A. Levy, "Two-dimensional model for simulating shock-wave interaction with rigid porous materials," AIAA J. 41, 663-673 (2003).

${ }^{57}$ F. Meyer, "Topographic distance and watershed lines," Signal Process. 38, 113-125 (1994).

${ }^{58}$ G. Dimonte, D. Youngs, A. Dimits, S. Weber, M. Marinak, S. Wunsch, C. Garasi, A. Robinson, M. Andrews, P. Ramaprabhu, et al., "A comparative study of the turbulent Rayleigh-Taylor instability using high-resolution three-dimensional numerical simulations: the alpha-group collaboration," Phys. Fluids 16, 1668-1693 (2004). 